The Astrophysical Journal, 681:856-880, 2008 July 10

(C) 2008. The American Astronomical Society. All rights reserved. Printed in U.S.A.

\title{
A POPULATION OF FAINT EXTENDED LINE EMITTERS AND THE HOST GALAXIES OF OPTICALLY THICK QSO ABSORPTION SYSTEMS ${ }^{1}$
}

\author{
Michael RaUCH \\ Observatories of the Carnegie Institution of Washington, 813 Santa Barbara Street, \\ Pasadena, CA 91101; mr@ociw.edu \\ Martin Haehnelt \\ Institute of Astronomy, Madingley Road, Cambridge CB3 0HA, UK; haehnelt@ast.cam.ac.uk \\ ANDREw BunKer \\ School of Physics, Stocker Road, Exeter EX4 4QL, UK; bunker@astro.ex.ac.uk \\ George Becker \\ Observatories of the Carnegie Institution of Washington, 813 Santa Barbara Street, \\ Pasadena, CA 91101; gdb@ociw.edu \\ Francine Marleau \\ Spitzer Science Center, Caltech, Mail Stop 220-6, 1200 East California Boulevard, \\ Pasadena, CA 91125; marleau@ipac.caltech.edu \\ JAMES GRAHAM \\ 601 Campbell Hall, University of California, Berkeley, CA 94720-3411; jrg@graham.berkeley.edu \\ Stefano CRistiani \\ Osservatorio Astronomico di Trieste, INAF, Via Tiepolo 11, 34143 Trieste, Italy; cristiani@oats.inaf.it \\ MATT JARVIS \\ Centre for Astrophysics, Science and Technology Research Institute, University of Hertfordshire, \\ Hatfield AL10 9AB, UK; M.J.Jarvis@herts.ac.uk \\ Cedric Lacey \\ Institute for Computational Cosmology, Department of Physics, Durham University, South Road, \\ Durham DH1 3LE, UK; cedric.lacey@durham.ac.uk \\ SIMON MORRIS \\ Department of Physics, Durham University, South Road, Durham DH1 3LE, UK; simon.morris@durham.ac.uk \\ Celine Peroux \\ Observatoire Astronomique de Marseille-Provence, Traverse du Siphon-Les Trois Lucs, \\ 13376 Marseille, France; celine.peroux@oamp.fr \\ HuUb RÖTTGERING \\ Leiden Observatory, NL-2300 RA Leiden, The Netherlands; rottgeri@strw.leidenuniv.nl \\ AND \\ Tom Theuns \\ Department of Physics, Durham University, South Road, Durham DH1 3LE, UK; tom.theuns@durham.ac.uk \\ Received 2007 September 6; accepted 2007 November 7
}

\begin{abstract}
We have conducted a long-slit search for low surface brightness Ly $\alpha$ emitters at redshift $2.67<z<3.75$. A $92 \mathrm{hr}$ long exposure with the ESO VLT FORS2 instrument down to a $1 \sigma$ surface brightness detection limit of $8 \times 10^{-20} \mathrm{erg} \mathrm{cm}^{-2}$ $\mathrm{s}^{-1} \operatorname{arcsec}^{-2}$ per arcsec ${ }^{2}$ aperture yielded a sample of 27 single line emitters with fluxes of a few $\times 10^{-18} \mathrm{erg} \mathrm{s}^{-1} \mathrm{~cm}^{-2}$. We present arguments that most objects are indeed Ly $\alpha$. The large comoving number density, $3 \times 10^{-2} h_{70}^{3} \mathrm{Mpc}^{-3}$, the large covering factor, $d N / d z \sim 0.2-1$, and the often extended Ly $\alpha$ emission suggest that the emitters can be identified with the elusive host population of damped Ly $\alpha$ systems (DLAS) and high column density Lyman limit systems (LLS). A small inferred star formation rate, perhaps supplemented by cooling radiation, appears to energetically dominate the Ly $\alpha$ emission, and is consistent with the low metallicity, low dust content, and theoretically inferred low masses of DLAS, and with the relative lack of success of earlier searches for their optical counterparts. Some of the line profiles show evidence for radiative transfer in galactic outflows. Stacking surface brightness profiles, we find emission out to at least $4^{\prime \prime}$. The centrally concentrated emission of most objects appears to light up the outskirts of the emitters (where LLS arise) down to a column density where the conversion from UV to Ly $\alpha$ photon becomes inefficient. DLAS, high column
\end{abstract}

\footnotetext{
${ }^{1}$ Based partly on observations made with ESO Telescopes at the Paranal Observatories under Program ID LP173.A-0440, and partly on observations obtained at the Gemini Observatory, which is operated by the Association of Universities for Research in Astronomy, Inc., under a cooperative agreement with the NSF on behalf of the Gemini partnership: the National Science Foundation (United States), the Science and Technology Facilities Council (United Kingdom), the National Research Council (Canada), CONICYT (Chile), the Australian Research Council (Australia), CNPq (Brazil) and CONICET (Argentina).
} 
density LLS, and the emitter population discovered in this survey appear to be different observational manifestations of the same low-mass, protogalactic building blocks of present-day $L_{*}$ galaxies.

Subject headings: diffuse radiation — galaxies: formation — intergalactic medium quasars: individual ([HGO96] DMS 2139.0-0405)

\section{INTRODUCTION}

Perhaps surprisingly, modern astronomy has found ways to study the material structures of the high-redshift universe over their entire vast range of densities and sizes, from the underdense large voids, via bright star-forming galaxies, to the most luminous QSOs.

The dark stages of this sequence, with densities from the universal mean to virial density, too low to produce detectable radiation, have been studied mainly with QSO absorption lines. This approach has taught us where to find most of the baryons (in the ionized Ly $\alpha$ forest gas; e.g., Rauch et al. 1997a) and most of the neutral gas (in damped Ly $\alpha$ systems; e.g., Wolfe et al. 1995) at high redshift. From the bright end of the cosmic matter distribution, serious inroads into the population of high-redshift galaxies have been made by color-selecting stellar continuum emitters, exploiting the Lyman limit continuum decrement (e.g., Steidel \& Hamilton 1993), or by searching for Ly $\alpha$ line emission induced by star formation (e.g., Cowie \& Hu 1998). The advent of spacebased, broadband galaxy surveys, in particular the Hubble Ultra Deep Field (Bunker et al. 2004; Beckwith et al. 2006; Bouwens et al. 2007), has begun filling in the gap between the essentially dark, lower mass range of subgalactic or barely star-forming protogalactic objects probed by absorption lines, and bright highredshift galaxies with large star formation rates of tens of $M_{\odot} \mathrm{yr}^{-1}$ (e.g., Erb et al. 2006).

The boundary between dark and bright is delineated by the important astrophysical transition from ionized gas to neutral, selfshielded gas, on a galactic mass scale. The underlying objects in which this transition has happened, known from absorption studies as Lyman limit systems (LLS), or, at higher column densities, damped Ly $\alpha$ systems (DLAS), have been rather elusive. We know quite a bit about their chemistry and ionization state, but little in terms of stellar contents, size, kinematics or mass.

Theoretical studies over the past two decades have suggested that a survey of LLS and DLAS for H I Ly $\alpha$ line emission of sufficient depth may uncover several distinct astrophysical sources of line emission that have the potential to shed considerable light (literally) on the distribution of neutral hydrogen, and thus on the bedrock of galaxy formation. If we acknowledge that high-redshift DLAS must have something to do with stars (they contain the main reservoir of neutral gas, and have a median metallicity of $[\mathrm{Z} / \mathrm{H}]=$ -1.5 , more than an order of magnitude higher than the coeval abundances in the intergalactic medium), then we may expect the potentially strongest signal to be star-formation-induced Ly $\alpha$ and/or a stellar continuum. Attempts to identify individual LLS or DLAS with galaxy counterparts have often been frustrated by the difficulty of detecting an extremely faint object (the DLAS host) next to an extremely bright object (a QSO). At low redshift $(z<1)$, where we can hope to learn most about the underlying galaxy population, observations have shown that DLA host galaxies represent a range of galaxy types (e.g., LeBrun et al. 1997; Chen et al. 2005), dominated by faint objects (e.g., Chun et al. 2006) with low star formation rates (Wild et al. 2007). Searches at high redshift (e.g., Warren et al. 2001; Fynbo et al. 2003; Kulkarni et al. 2000, 2001; Christensen et al. 2007) have so far produced only a handful of confirmed detections of the under- lying galaxies (Weatherley et al. 2005). Such efforts indicated that DLAS hosts at high redshift are generally drawn from the very faint end (Fynbo et al. 1999; Bunker et al. 1999b) of the general galaxy population at high redshift, intersected by the QSO line of sight at small impact parameters (Møller et al. 2002). Wolfe $\&$ Chen (2006) have recently performed a search for spatially extended continuum emission down to very faint levels using the Hubble Ultra Deep Field, and were able to place stringent upper limits on extended star formation in DLAS.

These results agree well with theoretical CDM-based models of galaxy formation (Kauffmann 1996) that envisage DLAS hosts as numerous small, faint, low-mass, merging protogalactic clumps (Haehnelt et al. 1998, 2000; Ledoux et al. 1998; Johansson \& Efstathiou 2006; Nagamine et al. 2007), rather than the large hypothetical disks (Prochaska \& Wolfe 1997) once popular.

Cooling radiation from collapsing galaxies (e.g., Haiman \& Rees 2001; Haiman et al. 2000; Fardal et al. 2001; Furlanetto et al. 2005) is a second process able to produce line radiation at fluxes competitive with those from star formation, at least in the halos of relatively massive galaxies (Dijkstra et al. 2006). The peculiar spatial distribution of the radiation and certain asymmetries of the spectral line profile, together with the local absence of a stellar continuum, could conceivably help to distinguish this source of radiation from star formation. The few candidates for such galactic cooling flows reported so far (Keel et al. 1999; Steidel et al. 2000; Francis et al. 2001; Bower et al. 2004; Dey et al. 2005; Matsuda et al. 2006; Nilsson et al. 2006; Smith \& Jarvis 2007) have been atypical for the galaxy population as a whole.

Perhaps the most curious of these emission processes is Ly $\alpha$ fluorescence, where $\mathrm{H}$ I ionizing photons impinging on optical thick gas are absorbed and converted with high efficiency to Ly $\alpha$ line radiation, albeit at a very low surface brightness level $\left(<10^{-19} \mathrm{erg} \mathrm{cm}^{-2} \mathrm{~s}^{-1} \operatorname{arcsec}^{-2}\right.$; Hogan \& Weymann 1987; Binette et al. 1993; Gould \& Weinberg 1996; Cantalupo et al. 2005). Lyman limit patches should light up the whole gaseous cosmic web to yield a relatively uniform, very faint "glow," perhaps locally enhanced by the proximity of a QSO (e.g., Cantalupo et al. 2005, 2007). A number of increasingly deep searches for this effect at high redshift have been performed (Lowenthal et al. 1990; Bunker et al. 1998, 1999a), in pursuit of a repeatedly shrinking theoretically predicted signal. A detection, which at the same time would be a measurement of the general UV background, has so far eluded us. Most recently, a number of detections of the enhanced $\operatorname{Ly} \alpha$ fluorescence in the proximity of QSOs have been reported (Fynbo et al. 1999; Bunker et al. 2003; Weidinger et al. 2004, 2005; Adelberger et al. 2006; Cantalupo et al. 2007; J. Hennawi 2007, private communication), but understanding of the effect has been complicated by the large number of degrees of freedom in the properties and behavior of the QSO (e.g., orientation, opening angle of the beam, life time).

Thus, there is good reason to conduct a search for extremely low light level Ly $\alpha$ line emission at high redshift. While most previous Ly $\alpha$ surveys have been performed as narrowband imaging searches, it is clear that the largest contrast with the sky background and the largest redshift (=spatial) depth is obtained by (long) slit spectroscopy. Because of the small volume covered, 
a blind search is not a viable way to find objects as rare as Lyman break galaxies, which are hard to hit with a single randomly positioned slit. In contrast, the rate of incidence of LLS with neutral hydrogen column densities exceeding $N(\mathrm{H} \mathrm{I})>10^{19} \mathrm{~cm}^{-2}$ per unit redshift is approximately unity at redshift $\sim 3$ (e.g., Peroux et al. 2003), i.e., the objects essentially cover the sky, and there should be numerous hits in a single setting for a typical long-slit spectrograph. We anticipate seeing about 30 patches of emission on a $2^{\prime \prime}$ wide and $7^{\prime}$ long slit at redshift $\sim 3$ (e.g., Bunker et al. 1998). Searching over a redshift range near $z \sim 3$ achieves a reasonable comprise between avoiding the ravages of $(1+z)^{4}$ dimming and succumbing to the relatively poor blue performance of most low-resolution spectrographs worldwide. However, in principle it is desirable to go as far to the blue as possible. The UV background as the source of the Ly $\alpha$ fluorescence is not expected to drop dramatically down to at least redshift 2, whereas the atmospheric background as the dominant source of noise decreases rapidly toward the blue.

Prior to the current ESO VLT project, the most sensitive search for Ly $\alpha$ fluorescence off optically thick gas, in regions dominated by the general UV background, had been performed by the long-slit experiment of Bunker et al. (1998, 1999a). The first attempt to measure this effect with the ESO VLT originated in a (shelved) science verification project for FORS, later submitted unsuccessfully during periods 64-66 as a large project (PI: Rauch). The amount of exposure time required even with an $8 \mathrm{~m}$ class telescope considerably exceeds typical observing time awards, so the strategy was to exploit the newly available service-mode observing and insert the observations during bad seeing periods, as the targets were expected to be extended, with radii of several arcseconds. The current incarnation, a combination of the two projects, reobserving the original target field of Bunker et al. (1999a), was awarded $120 \mathrm{hr}$ as ESO Large Project (LP173.A-0440: PI: Haehnelt). A parallel attempt to use the Gemini GMOS instruments on the same field chosen for the ESO project led to time awards with both Gemini telescopes (GN 2004-A-Q-91, GN-2005-B-Q-52, GS-2004-B-Q-61, GS2005-B-Q-36; PI: Bunker; and GN-2004-B-Q-35, GN-2004B-Q-35, GS-2004A-Q-78, GS-2004-B-Q-8: PI: Rauch). The Gemini effort resulted in $46 \times 3000 \mathrm{~s}$ exposures, of which 30 where taken with Gemini-North, 16 with Gemini-South. The Gemini data were taken at lower resolution (to take advantage of the most commonly used spectroscopic setup), but cover a larger redshift path than the ESO data. The current paper deals with the ESO data set; we postpone a full analysis of all data to a future paper. However, the Gemini data in a preliminary reduction have been consulted here in order to check the reality of the emitters found in the ESO data set (see below).

The ESO FORS2 project resulted in (after overheads) $92 \mathrm{hr}$ of on-source exposure, finally reaching a $1 \sigma$ surface brightness detection threshold of $8 \times 10^{-20} \mathrm{erg} \mathrm{cm}^{-2} \mathrm{~s}^{-1} \operatorname{arcsec}^{-2}$ (measured in a $1 \operatorname{arcsec}^{2}$ aperture). This is remarkably close to our expected sensitivity $\left(6.6 \times 10^{-20} \mathrm{erg} \mathrm{cm}^{-2} \mathrm{~s}^{-1} \operatorname{arcsec}^{-2}\right.$ in $\left.120 \mathrm{hr}\right)$.

The experiment is described below. Section 2 details the observational setup, and the sensitivity and spectral resolution limits reached. Section 3 spells out the individual properties of the emitters found, and gives estimates of the sizes, number densities, flux distributions, and derived rates of incidence. Section 4 discusses the possible identification with low redshift galaxies, and $\S 5$ ponders the consequences if the emitters are predominantly Ly $\alpha$. Alternative origins of the Ly $\alpha$ photons (stellar, QSO-induced, cooling radiation) are discussed in $\S 6$, including a detailed discussion of some unusual line profiles found. Section 7 establishes a connection between the emitters and QSO
DLAS, followed by a brief discussion of Ly $\alpha$ fluorescence in $\S 8$ and the conclusions in $\S$ 9. An Appendix discusses the importance of slit losses.

\section{OBSERVATIONS}

The QSO [HOG96] DMS 2139-0405 $(z=3.32, V=20.805$; Hall et al. 1996) was observed during 2004-2006 with the VLT FORS2 low-resolution spectrograph and the volume-phased holographic grism $1400 \mathrm{~V}$. A $2^{\prime \prime}$ wide and about $453^{\prime \prime}$ long slit gave a spectral resolution of $\lambda / \Delta \lambda_{\mathrm{FWHM}}=1050$. The CCDs were read out in $2 \times 2$ binned mode, giving pixels with an extent of $0.252^{\prime \prime}$ along the slit and about $0.64 \AA$ in the dispersion direction. Thus, the spectral resolution FWHM is sampled by about 8 pixels. The spectrum on the detector ranges from 4457 to $5776 \AA$, with a midpoint at $5099 \AA$.

The slit was centered on the QSO and rotated by a position angle $-5.95^{\circ}$, to repeat the orientation of the earlier Keck LRIS observation by Bunker et al. (1999a), where the particular position angle was chosen to minimize intersecting bright foreground galaxies.

A total of 110 exposures were taken between 2004 May and 2006 August, giving a total integration time of $92 \mathrm{hr}$. The exposures were divided roughly evenly into three dither positions separated on the sky by $10^{\prime \prime}$. The data were reduced using a custom set of IDL routines. Individual exposures were biassubtracted and flat-fielded. The sky was then subtracted from each exposure using an optimal sky-subtraction technique based on Kelson (2003), whereby the sky counts are modeled as a function of wavelength and slit position without rectifying the original data. Object traces that were visible in a single $3000 \mathrm{~s}$ exposure were masked when fitting the sky. The reduced two-dimensional spectra from all exposures and both FORS2 CCDs was then combined into single array with spectral dispersion closely matching the original exposures. Nearest-neighbor sampling was used when combining the frames to avoid correlating adjacent pixels. In order to avoid false detections, hot and cold pixels, bad rows, charge traps, and other defects were identified in the flat fields and reduced frames and aggressively excluded when producing the combined spectrum. Pixels with significant dark current, identified by combining large numbers of reduced exposures, were also rejected. In total, roughly $2.5 \%$ of the illuminated area of each CCD was masked when producing the combined spectrum. Combinations of various subsamples of the data were also made to check that no spurious features remained.

The resulting two-dimensional spectrum is shown in Figure 1. A flux calibration using several spectrophotometric standard stars reproduced the published flux of the QSO to within about $20 \%$. In the center of the final, sky-subtracted spectrum, a flux density of $1.6 \times 10^{-20} \mathrm{erg} \mathrm{cm}^{-2} \mathrm{~s}^{-1} \AA^{-1}$ produced 1 ADU per $0.252^{\prime \prime} \times 2^{\prime \prime} \times 0.64 \AA$ wide pixel. The observed standard deviation near the center of the spectrum is $2.0 \mathrm{ADU}$. We can reconstruct a surface brightness profile of a line emitter by integrating over the line in the spectral direction (over one FWHM, $\sim 286 \mathrm{~km} \mathrm{~s}^{-1}$ ). This gives a $1 \sigma$ surface brightness detection limit of $8.1 \times$ $10^{-20} \mathrm{erg} \mathrm{cm}^{-2} \mathrm{~s}^{-1} \operatorname{arcsec}^{-2}$, if measured in a $1 \operatorname{arcsec}^{2}$ aperture.

The "seeing" profile as measured off the QSO near the center of the combined final spectrum is 1.07 " FWHM. The seeing conditions generally were not as bad as anticipated, with $89 \%$ of the seeing better than $1.5^{\prime \prime}$.

The large number of continuum sources on the slit, the presence of a few brighter stars and galaxies with PSF wings visible at large distances, together with charge transfer and other cosmetic problems, exacerbated by a finite number of dithering positions, made a selection of objects with an automatic method 


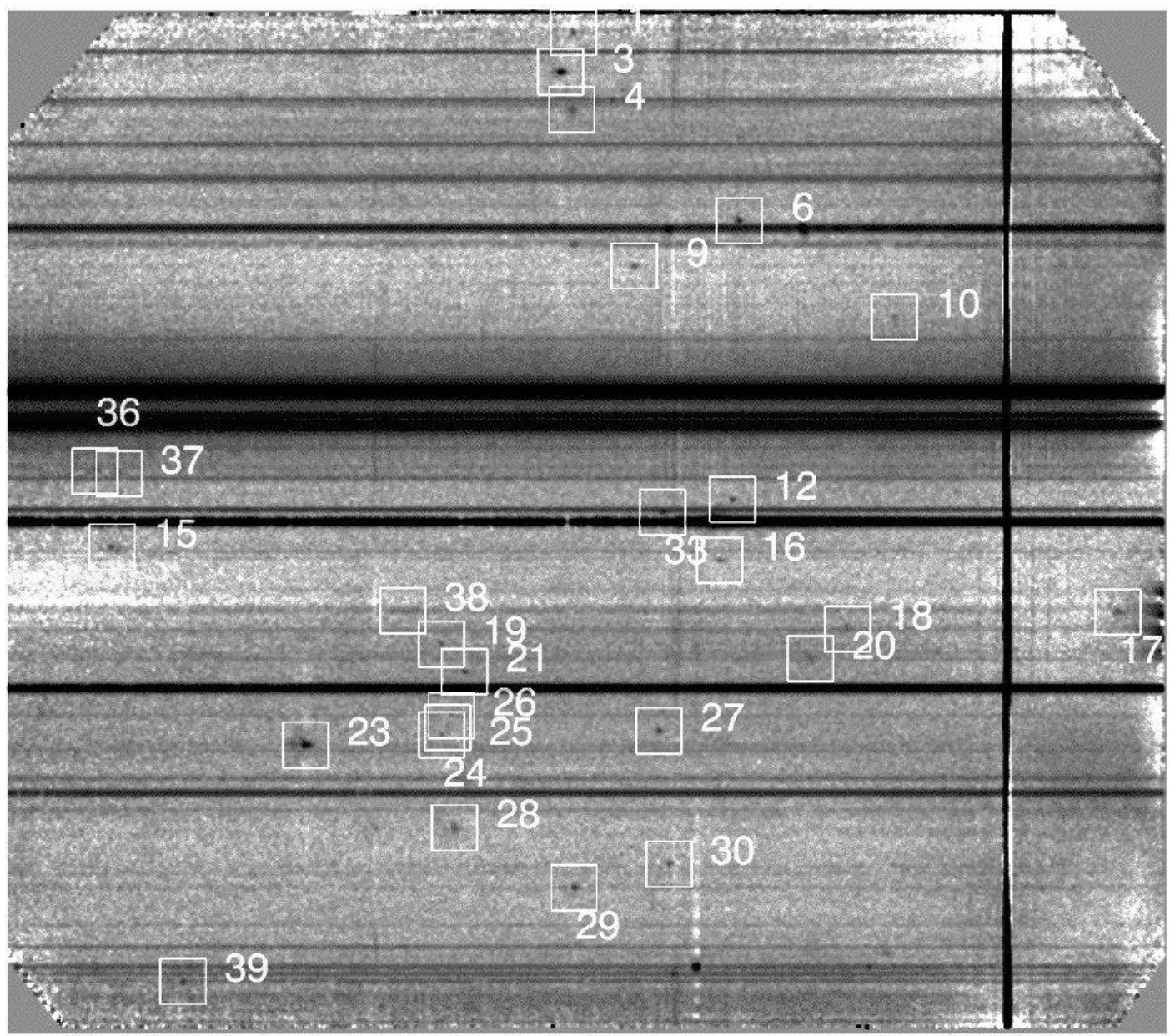

FIG. 1.- Two-dimensional spectrum obtained in $92 \mathrm{hr}$ of exposure time, showing the line emitter candidates for H I Ly $\alpha$ (boxes). The dispersion direction is hori-

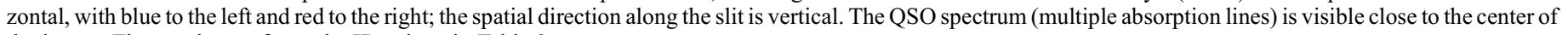
the image. The numbers refer to the IDs given in Table 2.

impractical. Thus, emission-line objects were selected by eye. The selected depth clearly varies with the character of the object (extended or point sources), but we estimate that for an extended object (angular extent $>1 \operatorname{arcsec}^{2}$ ) we get a visual detection at a surface brightness $2 \times 10^{-19} \mathrm{erg} \mathrm{cm}^{-2} \mathrm{~s}^{-1} \operatorname{arcsec}^{-2}$, reflected in the dearth of objects with a central surface brightness below that value in Figure 1. Quantitatively, this corresponds to about 3 standard deviations in the more precise surface brightness detection threshold based on pixel noise given above.

We have tested the reality of the emitters found by splitting the sample into halves. We were able to cross-identify all but two objects (numbers 18 and 25; see below) between the halves. One more (relatively bright) object (ID 2) was entirely absent in one half, but had already been excluded from the original sample because of its suspicious shape. Perusing the combined spectrum from the Gemini telescopes, we are able to cross-identify 9 of the 22 ESO objects of our emitter sample with common coverage by both the ESO and GMOS slits. However, most objects are barely detected in the shallower Gemini data.

The list in Table 2 contains 4 objects with a significance (based on the standard deviation of the flux) of less than $4 \sigma$. Of these, object 18 may or may not be real. Objects 19 and 26 certainly are present in both halves of the data set. Object 25 would not have been considered a detection everywhere in the twodimensional spectrum, but struck the eye because it seemed to constitute a group with the nearby objects 24,26 , and possibly 19 and 21 . Thus, we estimate that $\sim 2$ objects may be spurious detections. On the other hand, there are also a couple of candidates which could have been included in the sample but were not.

\section{OBSERVATIONAL RESULTS}

Spurious detection is a less serious concern than misidentification and the resulting contamination of the emitter sample by foreground low redshift galaxies, as lower redshift [O II] $3727 \AA$ (a doublet marginally resolved at our resolution), [O III] $5007 \AA$, or the $\mathrm{H}$ I Balmer series in emission could be mistaken for $\mathrm{H}$ I $\mathrm{Ly} \alpha$. We checked for the presence of these features and for others that could give away a low- $z$ object, such as the absence of a $\operatorname{Ly} \alpha$ forest decrement (when a continuum was present), and the existence of extended emission in the broadband image. Among the emission-line objects, five line emitters could be identified with foreground galaxies. They are listed in Table 1 and shown in Figure 2. The table gives the ID numbers, redshifts, the skysubtracted flux $F$ in units of $10^{-18} \mathrm{erg} \mathrm{cm}^{-2} \mathrm{~s}^{-1}$ measured in a $2^{\prime \prime} \times 2^{\prime \prime} \times 755 \mathrm{~km} \mathrm{~s}^{-1}$ aperture, the maximum surface brightness along the slit in units of $10^{-18} \mathrm{erg} \mathrm{cm}^{-2} \mathrm{~s}^{-1} \operatorname{arcsec}^{-2}$, and the source of the identification as a foreground galaxy. Since we were interested only in line emitters, we ignored the continuumonly objects for the time being. Three of the emission-line objects accidentally on the slit were identified with foreground galaxies based on the presence of [O II] (two spatially almost coincident objects at $z=0.39278$ and 0.4336 , with IDs $7 \mathrm{a}$ and $7 \mathrm{~b}$; and a 
TABLE 1

Foreground Emission Line OBJects

\begin{tabular}{|c|c|c|c|c|c|}
\hline $\begin{array}{l}\text { No. } \\
\text { (1) }\end{array}$ & $\begin{array}{l}\text { ID } \\
\text { (2) }\end{array}$ & $\begin{array}{c}z \\
(3)\end{array}$ & $\begin{array}{c}F\left[10^{-18}\right]^{\mathrm{a}} \\
(4)\end{array}$ & $\begin{array}{l}S_{\max }\left[10^{-18}\right] \\
\quad(5)\end{array}$ & $\begin{array}{l}\text { Source of Identification } \\
\text { (6) }\end{array}$ \\
\hline 1 & $7 \mathrm{a}$ & 0.3928 & $12.45 \pm 0.29$ & $5.06 \pm 0.22$ & $\mathrm{O}_{\text {II }}$ doublet \\
\hline $2 \ldots \ldots \ldots \ldots \ldots \ldots \ldots \ldots \ldots$ & $7 \mathrm{~b}$ & 0.4336 & $27.53 \pm 0.33$ & $8.99 \pm 0.23$ & O II doublet \\
\hline $3 \ldots \ldots \ldots \ldots \ldots \ldots \ldots \ldots \ldots \ldots \ldots$ & 8 & 0.0458 & $2.60 \pm 0.28$ & $1.10 \pm 0.21$ & $\mathrm{H} \beta$ and $\mathrm{Mg} b$ triplet \\
\hline 4 & 22 & 0.1980 & $18.49 \pm 0.31$ & $8.30 \pm 0.23$ & Balmer lines, $\mathrm{O}_{\text {II }}$ doublet \\
\hline 5 & 31 & 0.4019 & $26.55 \pm 0.32$ & $9.24 \pm 0.23$ & O II doublet \\
\hline
\end{tabular}

${ }^{\text {a }}$ Total flux for both lines where doublet.

galaxy at $z=0.4019$, ID 31). A fourth object appears to show an $[\mathrm{O}$ II] doublet on the very edge of the detector, and is clearly identified by several H I Balmer emission lines as a $z=0.198$ galaxy (ID 22). A fifth object shows $\mathrm{H} \beta$ and the $\mathrm{Mg} b$ triplet at $z=0.0458$ (ID 8). The contamination of the remaining sample of emitters by [O II] and [O III] is still a concern, because we do not have information about the continuum for most objects. We will treat the sample of emitters formally as $\operatorname{Ly} \alpha$ in most of the paper, but will return to a discussion of contamination below. The remaining 27 line emitters are listed in Table 2.

Figure 3 shows the two-dimensional spectra of all remaining candidate Ly $\alpha$ emission-line regions. Numbers correspond to the ID (col. [2] in Table 2). The "stamps" are $60 \times 60$ pixels wide, with an individual pixel size of $0.252^{\prime \prime} \times 0.64 \AA$. Thus, each stamp corresponds to $15.12^{\prime \prime}$ (116 physical kpc at the central redshift, $z=3.2$ ) in the spatial direction (vertical) and $38.4 \AA\left(2266 \mathrm{~km} \mathrm{~s}^{-1}\right.$ at the CCD center) in the dispersion direction (horizontal; wavelength increases toward the right). The spectra have been heavily smoothed in both the spectral and spatial direction (with a $7 \times 7$ boxcar filter) for display purposes and to emphasize coherent regions of extended emission. All spectra are displayed to within the same color stretch to demonstrate the variety in their appearance. Pixels within the light gray areas correspond to a flux density $>1.5 \times 10^{-20} \mathrm{erg} \mathrm{cm}^{-2} \mathrm{~s}^{-1} \AA^{-1}$.

A close-up of the spectra, showing $7.56^{\prime \prime}$ by $25.6 \AA$ in a less highly smoothed $(3 \times 3$ pixels $)$ version is seen in Figure 4 . Here the color stretch was done individually for each image to emphasize the individual dynamic range. The QSO Ly $\alpha$ emission-line region (ID 14) is also shown for reference, but was not included in the actual analysis. Next to the two-dimensional spectra are the object IDs, and a background-subtracted one-dimensional spectrum produced by collapsing the box along the slit direction (vertical) in units of erg s $\mathrm{cm}^{-1} \mathrm{~cm}^{-2} \AA^{-1}$, followed by a spatial surface brightness profile along the slit direction that was obtained by collapsing the box in the dispersion direction and averaging the bottom and top sections to improve the signal-to-noise ratio
$(\mathrm{S} / \mathrm{N})$. The velocity and spatial zero points are the left edge of the box and the center of the box, respectively, where the box is centered on the peak of a Gaussian fit to the spectral and spatial light profile.

The one-dimensional spectra often look poor, due to the faint signal and the box size, folding in a lot of noise, and sometimes wings of other objects. Optimal extraction has also been performed, but with surprisingly little improvement.

To help judge whether a surface brightness profile is extended, the spatial profiles to the right in Figure 4 also show a model fit, where the surface brightness $S(y)$ is represented by a Gaussian core plus power-law wings (dotted profile). First the Gaussian with the fixed width of the point-spread function (dashed profile) is fit to the innermost two pixels, to determine the amplitude parameter $A$ for the core of the emission. Then, beyond a distance $y_{t}$ along the slit from the center of the emission, a power law replaces the Gaussian. The transition distance along the slit from the center of the emission, $y_{t}$, and the power-law index are treated as free parameters:

$$
S(y)= \begin{cases}A \exp \left[-y^{2} /\left(2 \sigma^{2}\right)\right], & y<y_{t}, \\ A \exp \left[-y_{t}^{2} /\left(2 \sigma^{2}\right)\right]\left(y / y_{t}\right)^{\alpha}, & y>y_{t} .\end{cases}
$$

In one instance the spatial fit failed because there were other objects nearby.

Most of the objects seem to have a relatively well-defined emission peak, often surrounded by diffuse spatial emission, sometimes with rather broad emission lines (Fig. 3). We have tried to loosely classify these objects visually, according to whether they are consistent with being point sources (i.e., have a Gaussian seeing profile with a FWHM $\sim 1.07^{\prime \prime}$, as derived from the QSO), labeled as "PS" in Table 2, whether they are dominated by amorphous extended emission, labeled as "EXT," or a mix between the two, centrally dominated ("CD") emission yet with an extended halo around them. The distinction is often subjective,
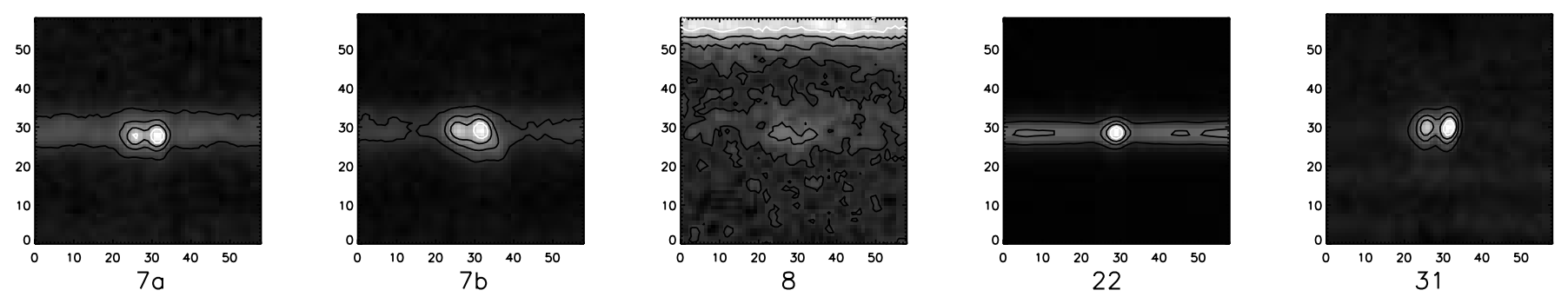

Fig. 2.- Spectra of the emission line regions of five foreground line-emitting galaxies identified from their $\mathrm{O}$ II doublet (objects 7a, 7b, 31) or Balmer emission ( $\mathrm{H} \beta$ for object $8, \mathrm{H} \gamma$ for object 22) features. The coordinates are in pixel units $\left(0.252^{\prime \prime} \times 0.67 \AA\right)$. The sections of the spectra shown here are $15.12^{\prime \prime}$ wide in the spatial direction and about $2266 \mathrm{~km} \mathrm{~s}^{-1}$ long in the spectral direction (i.e., horizontally). The numbers refer to the IDs given in Table 1. 
TABLE 2

Properties of Single-Line Emitters

\begin{tabular}{|c|c|c|c|c|c|c|c|c|c|c|}
\hline $\begin{array}{l}\text { No. } \\
\text { (1) }\end{array}$ & $\begin{array}{l}\text { ID } \\
\text { (2) }\end{array}$ & $\begin{array}{c}z \\
(3)\end{array}$ & $\begin{array}{c}F\left[10^{-18}\right] \\
(4)\end{array}$ & $\begin{array}{c}\text { Flux Ratio } \\
\text { (5) }\end{array}$ & $\begin{array}{c}S_{\max }\left[10^{-18}\right] \\
(6)\end{array}$ & $\begin{array}{l}v_{\text {FWHM }} \\
\text { (7) }\end{array}$ & $\begin{array}{c}A\left[10^{-18}\right] \\
(8)\end{array}$ & $\begin{array}{l}y_{t} \\
(9)\end{array}$ & $\begin{array}{c}\alpha \\
(10)\end{array}$ & $\begin{array}{l}\text { Comments }{ }^{\mathrm{a}} \\
\text { (11) }\end{array}$ \\
\hline $1 \ldots \ldots \ldots$ & 1 & 3.1801 & $2.32 \pm 0.57$ & $0.53 \pm 0.13$ & $0.88 \pm 0.4$ & 352.2 & 1.04 & 3.0 & -2.12 & somewhat EXT, CD \\
\hline $3 \ldots \ldots \ldots$. & 4 & 3.1797 & $3.38 \pm 0.34$ & $0.47 \pm 0.05$ & $1.14 \pm 0.24$ & 431.8 & 1.28 & 2.9 & -0.50 & EXT \\
\hline $4 \ldots \ldots \ldots$ & 6 & 3.3362 & $6.04 \pm 0.35$ & $0.67 \pm 0.04$ & $2.09 \pm 0.24$ & 289.8 & 2.26 & 3.9 & -2.12 & $\mathrm{CD}$ \\
\hline $5 \ldots \ldots \ldots$ & 9 & 3.2378 & $2.89 \pm 0.35$ & $0.79 \pm 0.10$ & $0.81 \pm 0.24$ & 288.3 & 0.92 & 2.2 & -3.78 & $\mathrm{CD}$, coincidence with unrelated (?) continuum object \\
\hline $8 \ldots \ldots \ldots$ & 14 & 3.3221 & & & & & & & & QSO centered on broad em. line \\
\hline $9 \ldots \ldots \ldots$ & 15 & 2.7682 & $2.67 \pm 0.41$ & $0.38 \pm 0.06$ & $1.02 \pm 0.29$ & 883.4 & 1.04 & 2.4 & -0.91 & EXT "ring" with blue-dominated double comp. \\
\hline $10 \ldots \ldots$. & 16 & 3.3189 & $3.06 \pm 0.37$ & $0.59 \pm 0.07$ & $1.08 \pm 0.28$ & 528.3 & 1.48 & 4.1 & -2.20 & $\mathrm{CD}$; broad em. line \\
\hline $11 \ldots \ldots$. & 17 & 3.6954 & $4.12 \pm 0.45$ & $0.62 \pm 0.07$ & $1.40 \pm 0.32$ & 493.9 & 1.48 & 2.7 & -1.68 & EXT; broad em. line \\
\hline $12 \ldots \ldots$. & 18 & 3.4373 & $1.14 \pm 0.37$ & $0.50 \pm 0.16$ & $0.48 \pm 0.27$ & 289.3 & 0.24 & 2.0 & -0.54 & EXT, amorphous \\
\hline $13 \ldots \ldots .$. & 19 & 3.0595 & $1.53 \pm 0.44$ & $3.27 \pm 0.94$ & $0.47 \pm 0.30$ & 158.8 & 0.13 & 3.1 & -2.20 & $\mathrm{CD}$, somewhat EXT \\
\hline $14 \ldots \ldots$. & 20 & 3.4023 & $1.79 \pm 0.37$ & $0.37 \pm 0.08$ & $0.68 \pm 0.26$ & 514.3 & 0.69 & 2.1 & -0.99 & EXT, amorphous \\
\hline $19 \ldots \ldots$. & 26 & 3.0692 & $-0.04 \pm 0.37$ & $-0.22 \pm 2.02$ & $0.19 \pm 0.25$ & 238.9 & fit & failed & & PS, faint \\
\hline $20 \ldots \ldots$. & 27 & 3.2617 & $3.51 \pm 0.34$ & $0.78 \pm 0.08$ & $1.23 \pm 0.24$ & 249.2 & 1.32 & 4.7 & -2.49 & PS; narrow line \\
\hline $21 \ldots \ldots$. & 28 & 3.0732 & $2.90 \pm 0.36$ & $0.45 \pm 0.06$ & $1.07 \pm 0.25$ & 885.7 & 1.09 & 2.2 & -1.09 & $\mathrm{CD}$, somewhat EXT; weak blue, strong red double comp. \\
\hline $22 \ldots \ldots$. & 29 & 3.1819 & $3.96 \pm 0.34$ & $0.48 \pm 0.04$ & $1.36 \pm 0.24$ & 279.5 & 1.57 & 3.3 & -0.34 & $\mathrm{CD}$; weak blue, strong narrow red double comp. \\
\hline $23 \ldots \ldots$. & 30 & 3.2715 & $3.05 \pm 0.35$ & $1.56 \pm 0.18$ & $1.27 \pm 0.25$ & 313.1 & 0.88 & 4.4 & -2.38 & $\mathrm{CD}$ \\
\hline $24 \ldots \ldots$. & 33 & 3.2646 & $3.50 \pm 0.34$ & $1.00 \pm 0.10$ & $1.88 \pm 0.24$ & 322.2 & 1.51 & 4.0 & -2.16 & PS; coincidence with unrelated lower $z$ continuum obj. \\
\hline $25 \ldots \ldots$. & 36 & 2.7483 & $3.46 \pm 0.52$ & $0.81 \pm 0.12$ & $0.90 \pm 0.31$ & 1073.7 & 1.08 & 2.7 & -1.62 & very EXT, amorphous smudge, nearby QSO and gal. abs. \\
\hline $26 \ldots \ldots$. & 37 & 2.7713 & $3.27 \pm 0.57$ & $1.14 \pm 0.20$ & $0.73 \pm 0.29$ & 725.0 & 1.02 & 4.9 & -2.76 & EXT, assym.; em. ("trapdoor”) blueward of abs. \\
\hline $27 \ldots \ldots$. & 38 & 3.0322 & $3.43 \pm 0.70$ & $2.69 \pm 0.55$ & $1.44 \pm 0.51$ & 2151.1 & 0.79 & 5.2 & -2.70 & very broad em. on top of fuzzy continuum \\
\hline $28 \ldots \ldots$. & 39 & 2.8285 & $2.28 \pm 0.43$ & $0.41 \pm 0.08$ & $0.75 \pm 0.33$ & 275.6 & 0.86 & 2.2 & -1.07 & $\mathrm{CD}$, narrow P Cygni em. line w. continuum \\
\hline
\end{tabular}

${ }^{\mathrm{a}} \mathrm{EXT}=$ extended $\mathrm{CD}=$ centrally dominated; $\mathrm{PS}=$ point source.

and is used only to introduce some nomenclature to aid the discussion. In this scheme, objects $1,4,9,10,15,16,17,18,20,28$, 36,37 , and 39 are clearly extended, objects $6,9,16,19,23,29$, and 30 could be classified as centrally dominated, and objects 3,12 , $21,24,25,26,27$, and 33 are in the somewhat better defined "point source" class.

Several among the brighter, mostly PS and CD sources (objects $3,23,27,28,29,39)$ exhibit the classic asymmetric line profiles known from previous studies of starbursting galaxies (e.g., Franx et al. 1997; Mas-Hesse et al. 2003; Tapken et al. 2007), with a blue cutoff and a more extended red wing. A subset of those (3, 23, 28, and 29) appear to have a weaker blue emission line in addition to the red dominant one, perhaps a sign of the double-humped emission profile expected from a static, externally illuminated slab (e.g., Neufeld 1990; Zheng \& Miralda-Escude 2002).

Intriguingly, at least one object (ID 15) shows the opposite situation, a stronger blue line opposed by a weaker red one, and there are two other bizarrely shaped objects $(36,37)$ in which the emission seems to occur blueward of the absorption the objects cause in a nearby galaxy continuum. In those two cases the emission seems to drift redward toward larger distances from the absorber. Object 38 shows a large emission region on top of a diffuse continuum, but because of its low $\mathrm{S} / \mathrm{N}$ remains unclassifiable.

The properties of the detected sources are listed in Table 2. Column (1) gives the number of the entry in the table, column (2) the identification number of each object, as used throughout the paper (including the figures); column (3) the redshift, assuming the emitter is Ly $\alpha 1215.67 \AA$; column (4) the sky-subtracted flux $F$ in units of $10^{-18} \mathrm{erg} \mathrm{cm}^{-2} \mathrm{~s}^{-1}$ measured in a $2^{\prime \prime} \times 2^{\prime \prime} \times 755 \mathrm{~km} \mathrm{~s}^{-1}$ aperture; column (5) the ratio of that flux to the one measured in a larger $\left(2^{\prime \prime} \times 7.6^{\prime \prime} \times 1510 \mathrm{~km} \mathrm{~s}^{-1}\right)$ aperture; column (6) the maximum surface brightness along the slit in units of $10^{-18} \mathrm{erg} \mathrm{cm}^{-2}$ $\mathrm{s}^{-1} \operatorname{arcsec}^{-2}$; column (7) the FWHM velocity width of a singlecomponent Gaussian fit to the optimally extracted emission line as a crude measure of overall velocity width, even where the line shape was distinctly non-Gaussian; column (8) the Gaussian amplitude of the central emission region for the surface brightness model profile described in the text; column (9) the turnover distance between Gaussian center and power law wings for that model; column (10) the power-law index for that model; and in column (11) objects are loosely classified as PS (point source), EXT (extended), or CD (centrally dominated), and peculiarities are noted.

The table shows a few instances where the ratio between the fluxes measured in the smaller and larger apertures was formally larger than unity. This happens when horizontal streaks on the CCD and sky residuals enter the larger window but not the small one, or when the background-subtraction windows were in different positions for the two apertures.

The errors quoted are standard deviations propagated in the usual way from the original pixel photon fluctuations. The $1 \sigma$ noise of the sky-subtracted flux (entry 4 ) is quoted for the $2^{\prime \prime} \times$ $2^{\prime \prime} \times 755 \mathrm{~km} \mathrm{~s}^{-1}$ aperture, the noise for the maximum surface brightness (entry 6) is per pixel. The $\mathrm{S} / \mathrm{N}$ attained is totally dominated by the sky background, with minor contributions from the detector noise and suppression of cosmic rays.

A histogram of the wavelength distribution, together with a plot of the relative sensitivity of the observation, is given in Figure 5. 


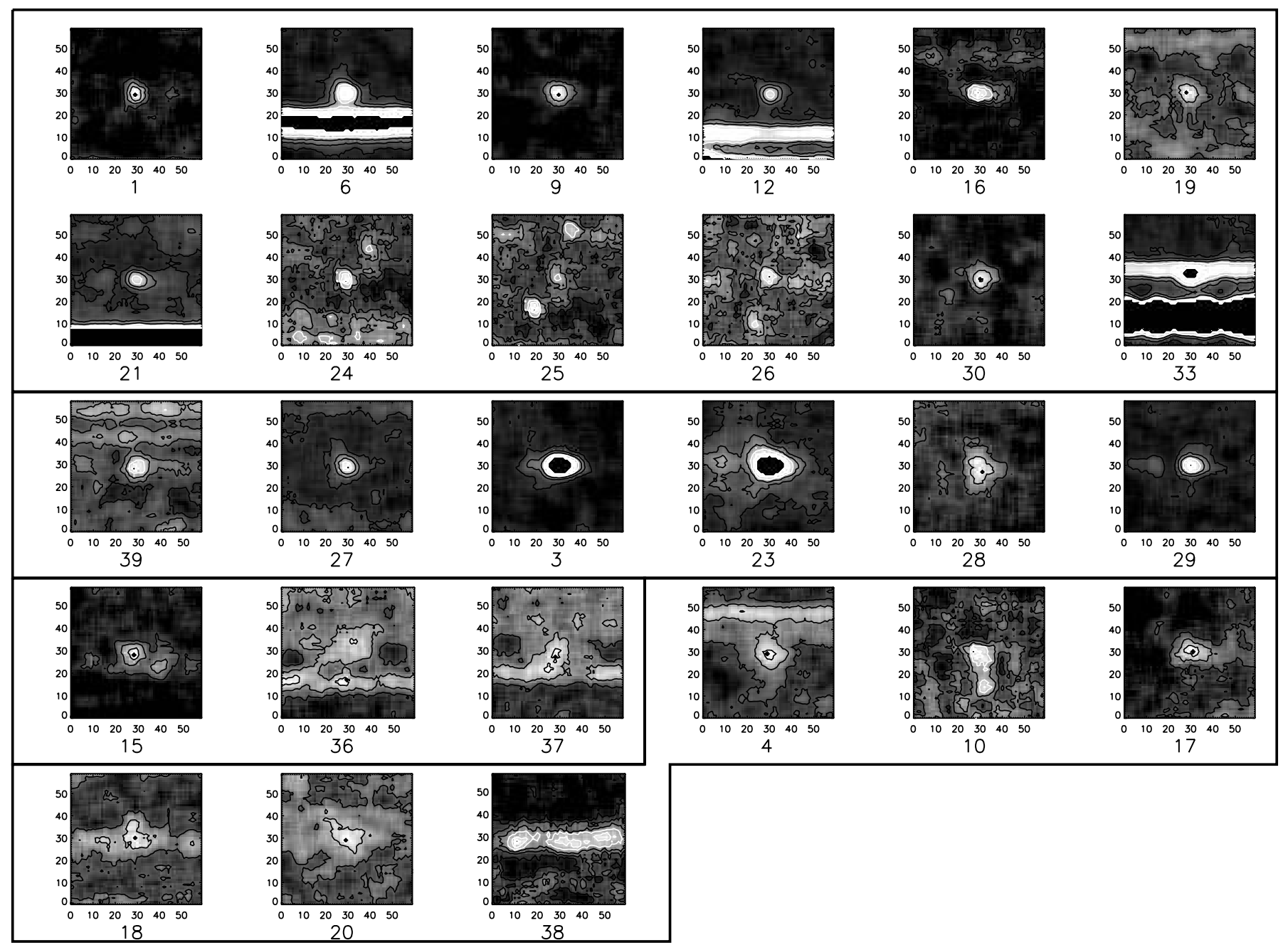

FIG. 3.-Short spectra for the single line emitters. The coordinates are in pixel units $\left(0.252^{\prime \prime} \times 0.67 \AA\right)$. The sections of the spectra shown here are $15.12^{\prime \prime}$ or 116 proper $\mathrm{kpc}$ wide in the spatial direction and about $2266 \mathrm{~km} \mathrm{~s}^{-1}$ long in the spectral direction (i.e., horizontally). The spectra have been heavily smoothed with a $7 \times 7$ pixel boxcar filter. The areas within the light gray contours have a flux density greater than approximately $1.5 \times 10^{-20} \mathrm{erg} \mathrm{cm}^{-2} \mathrm{~s}^{-1} \AA$. The numbers refer to the IDs given in Table 2 . The spectra are grouped together such that the first 12 (top box) appear to have a single central peak; the next six (IDs 39, 27, 3, 23, 28, and 29; second box from top) show a clearly asymmetric red peak, with a much weaker blue counterpeak; the following three (third box to the left) have either a stronger blue than red peak (ID 15) or emission features blueward of an absorption line $(36,37)$; the remaining six are unclassifiable, sometimes amorphous objects.

\subsection{Spatial Profiles}

The size of the emitters can be characterized by a contour at which the surface brightness has dropped to a particular level. We define somewhat arbitrarily a projected "size" along the slit as the distance $y$ from the center of the object along the slit at which the surface brightness of the fit model (1) has risen to $S(y)=1 \times 10^{-19} \mathrm{erg} \mathrm{cm}^{-2} \mathrm{~s}^{-1} \operatorname{arcsec}^{-2}$ (approximately the standard deviation in surface brightness in a $1 \operatorname{arcsec}^{2}$ aperture), going inward. This is close to the surface brightness level that corresponds to a $3 \sigma$ detection threshold in an opening given by the product of slit width $\left(2^{\prime \prime}\right)$ and FWHM (1.07"), i.e., it would be the approximate detection threshold for an unresolved source. By "size" we mean, in the following, half the total extent along the slit, analogous to the "radius" of an object. Note that this is generally an underestimate of the true radius. The two are only identical if the slit is centered on the emitter. The distribution of these "sizes" for our 27 objects is given in Figure 6.

Most of the sizes occur near the spatial resolution limit ( $\sim 0.54^{\prime \prime}$, HWHM ), but there is a considerable tail to much larger radii. Four large objects that clearly extend beyond our fitting range are collected in the bin at $3.6^{\prime \prime}$. This bin comprises objects $4,15,23$, and 29 . The median "radius" is 0.99 ", or 7.7 physical kpc for a Ly $\alpha$ emitter at redshift 3.2.

\subsection{Number Densities and Fluxes}

Some of our 27 emitters are unambiguously identifiable (as H I Ly $\alpha 1216 \AA$ ) just based on the line profile shapes. The same is true for the identification of the additional four bright double component objects as [O II] 3726, $3729 \AA$ emitters. Nevertheless, the low $\mathrm{S} / \mathrm{N}$ and the mostly invisible continuum do not permit us to a priori exclude the identification of the majority of the sources with either of those two classes of objects, $2.667<z<3.751$ Ly $\alpha$ or $0.196<z<0.550$ [O II]. The 16 reddest of the 27 sources are at least in the right redshift range $(0<z<0.16)$ to also be eligible for [O III] $5007 \AA$, as the weaker [O III] transition and $\mathrm{H} \beta$ usually would be too faint to be seen. Although these possibilities are not equally likely, as we shall argue below, it is instructive to look at the implied number densities, luminosity functions, and fluxes for the three extreme interpretations. 

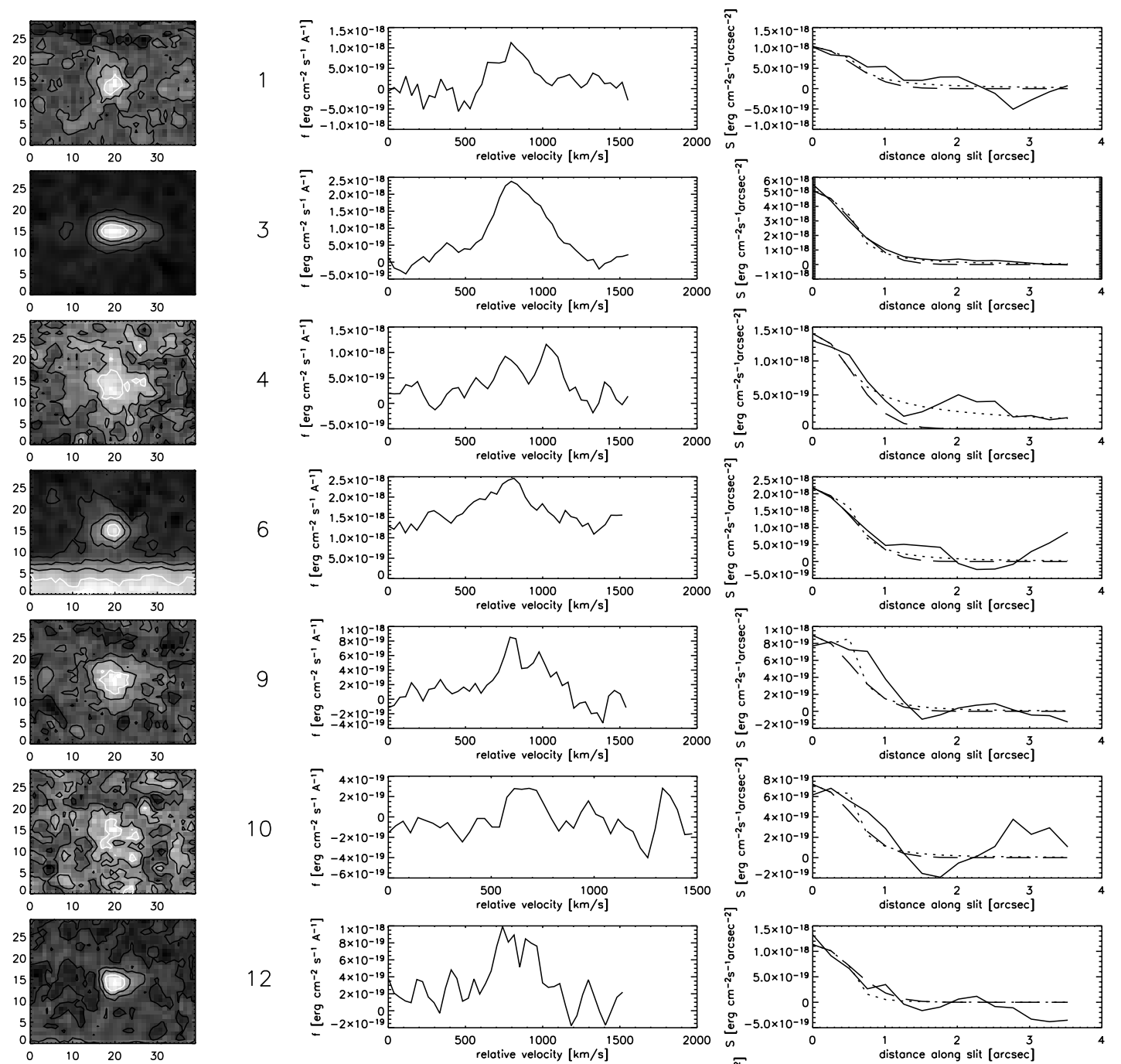

4

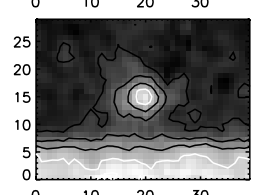

6
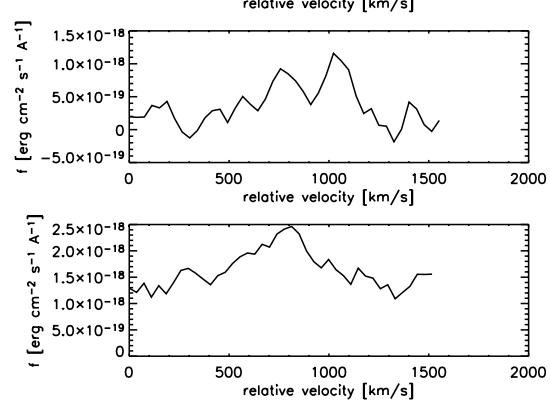

9

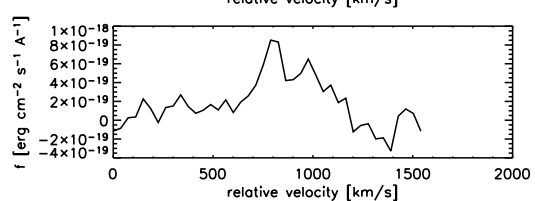

10
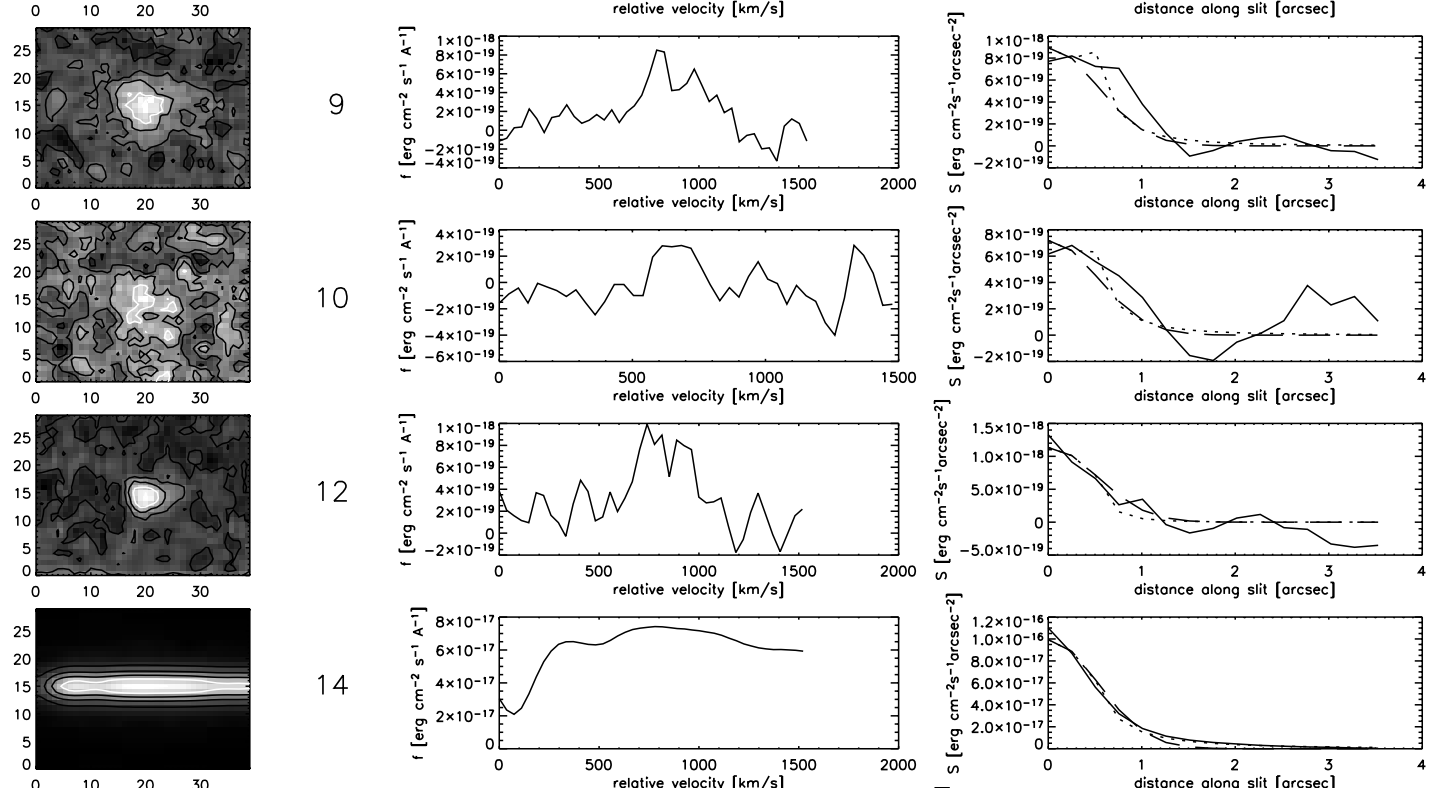

12

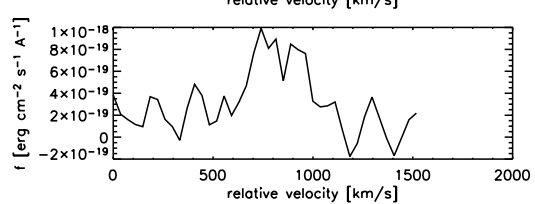

14
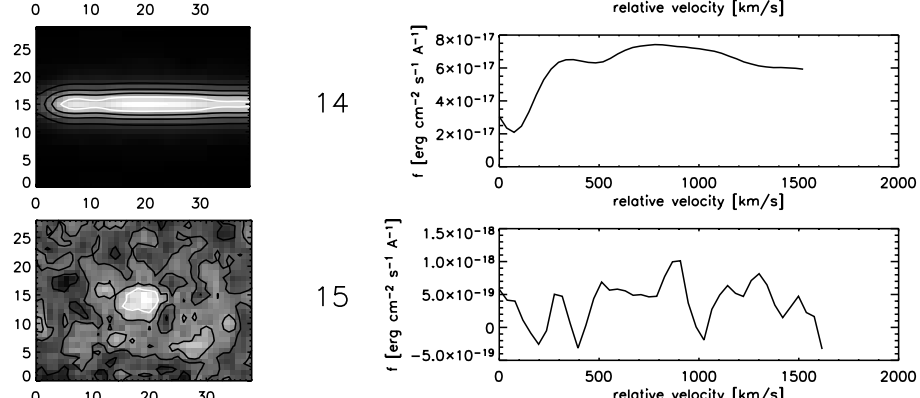

15
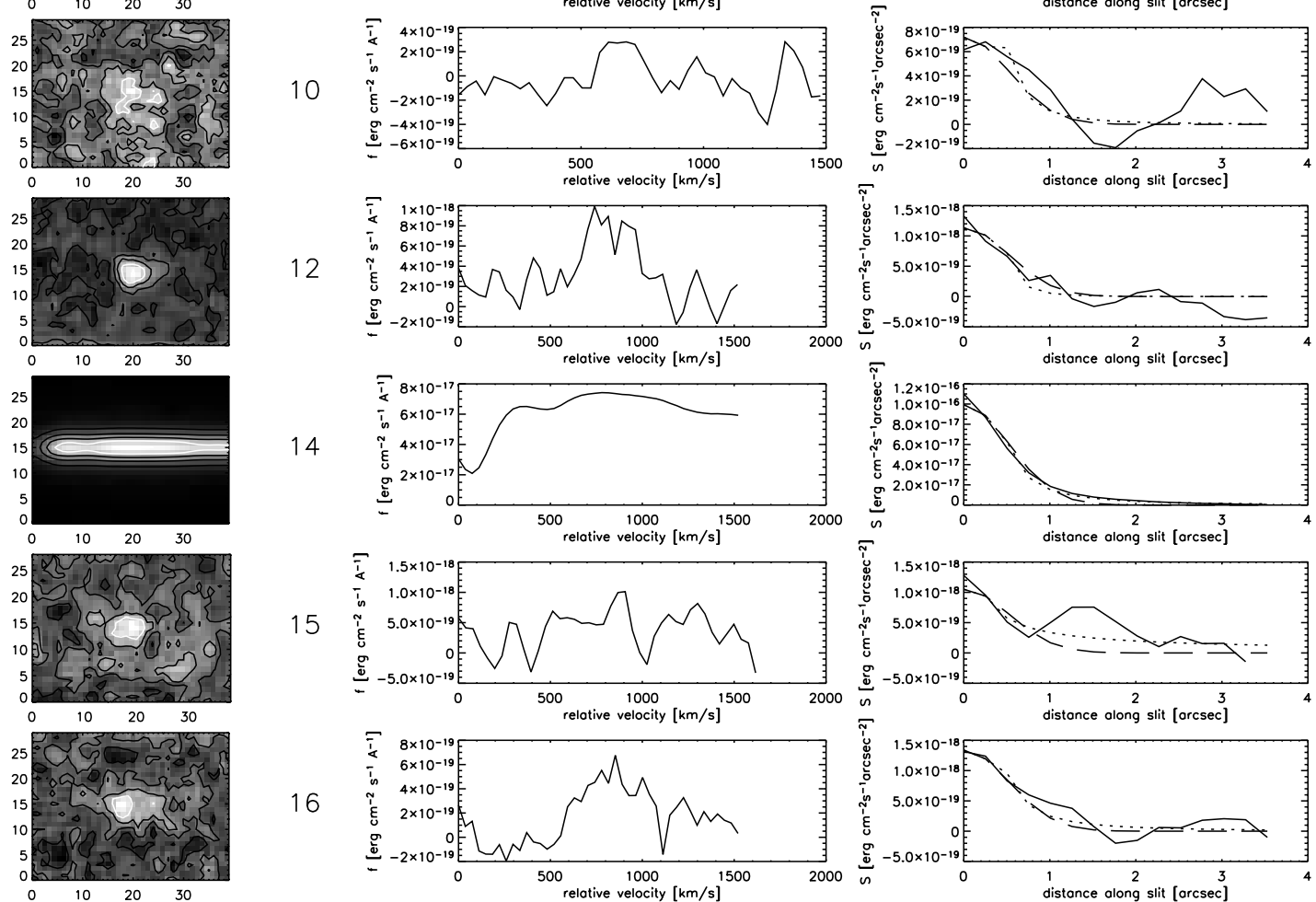

FIG. 4.- Two-dimensional spectra, extracted one-dimensional spectra (flux density in erg $\mathrm{cm}^{-2} \mathrm{~s}^{-1} \AA^{-1}$ ), and spatial surface brightness cross sections (in erg cm ${ }^{-2}$

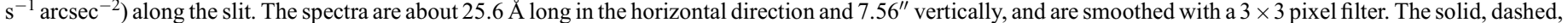

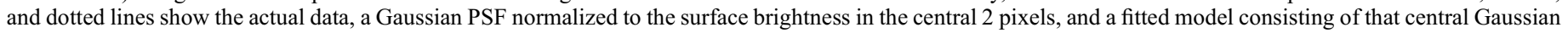
and a power-law continuation farther out, respectively. 

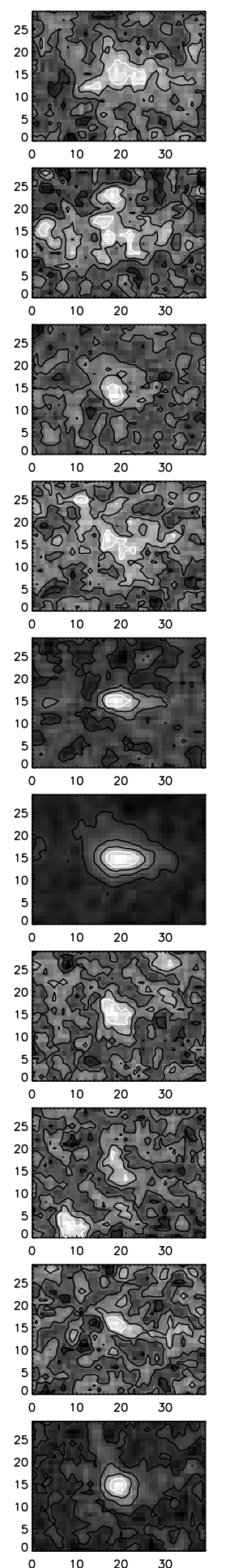

17

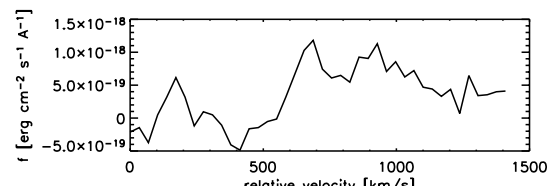

18
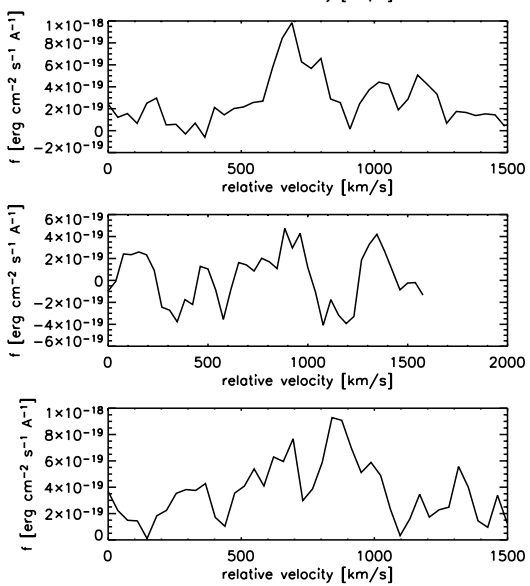

$\bar{i} 8 \times 10^{-19}$
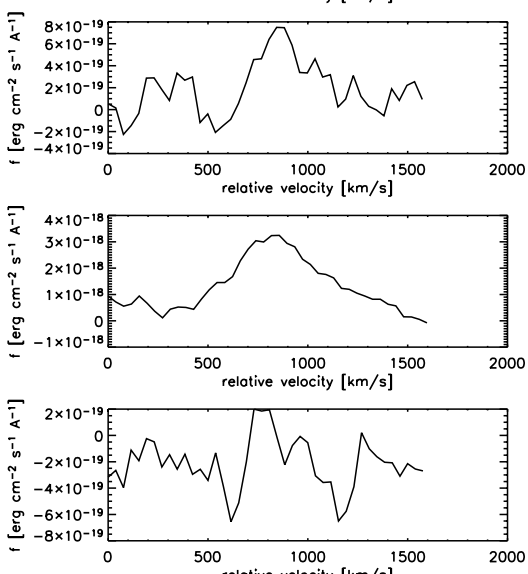$$
500 \quad 1000 \quad 1500 \quad 2000
$$
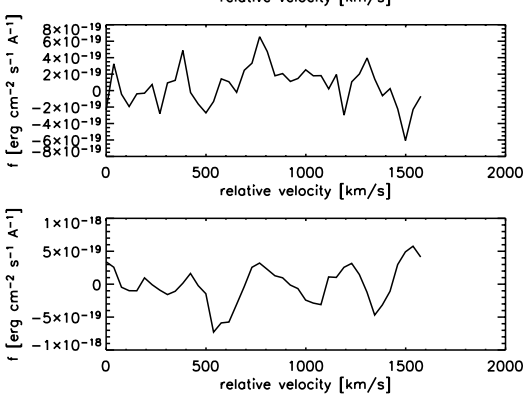

27
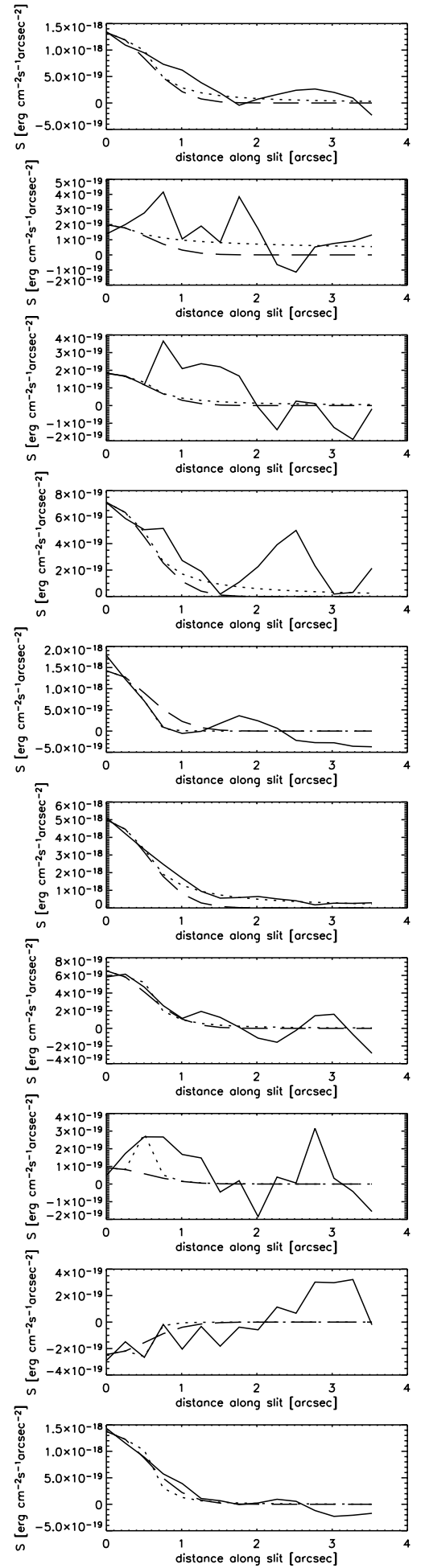

FIG. 4-Continued 

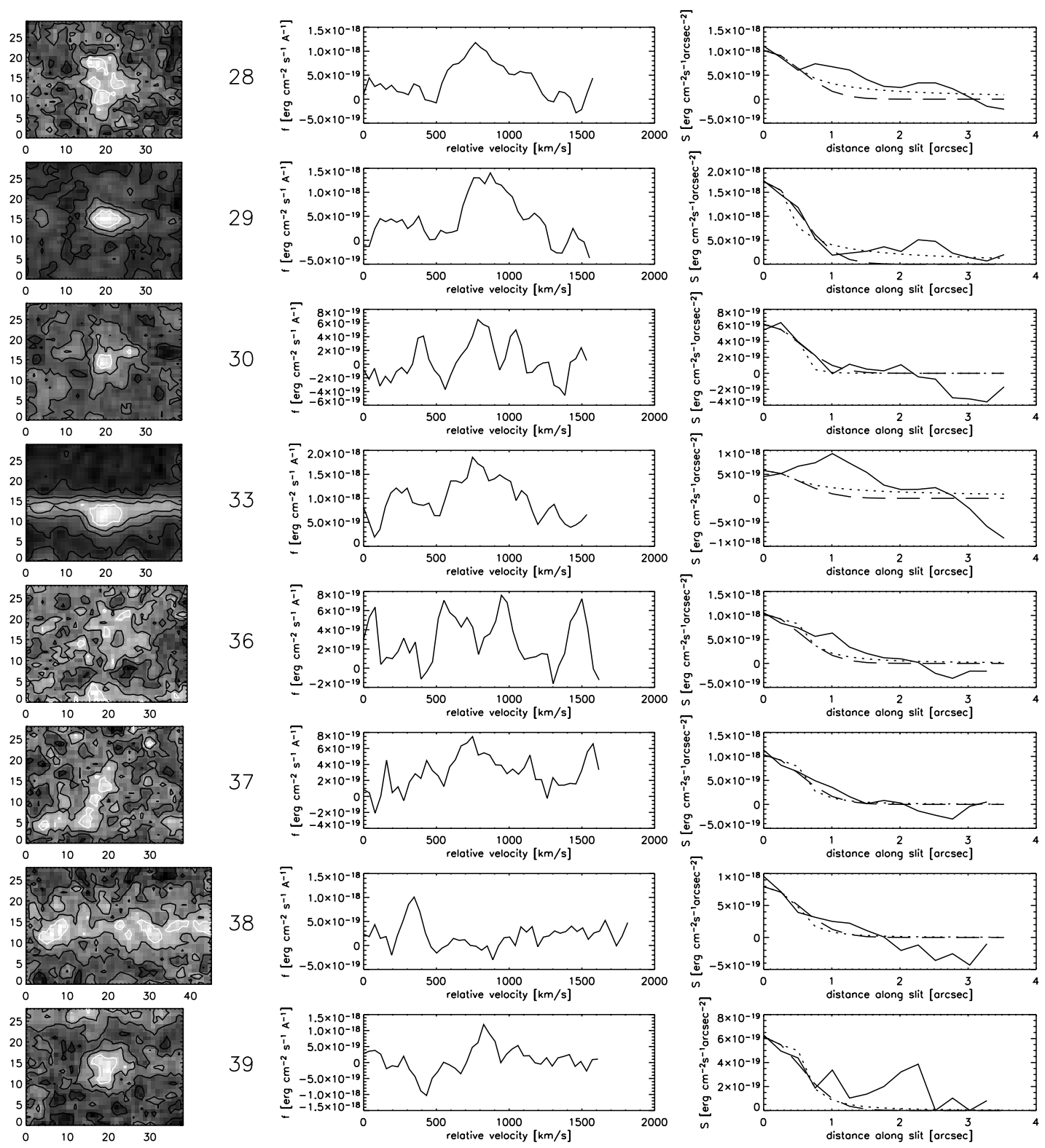

Fig. 4-Continued

For the purposes of this paper we adopt a flat cosmology with $\Omega_{m}=0.3, \Omega_{\Lambda}=0.7$, and $H_{0}=70 \mathrm{~km} \mathrm{~s}^{-1} \mathrm{Mpc}^{-1}$. For the following analysis we have not applied upward corrections for slit losses to the luminosities, which occur when an object is larger than the slit. These corrections can be important, but are generally uncertain for a population of objects with an apparently large range of sizes and profile shapes. For a detailed discussion of slit losses, we refer the reader to the Appendix. We emphasize here that the luminosities for objects with characteristics encountered in our sample may be underestimated by up to factors of 2-5.

The redshift range of the spectrum, assuming $\mathrm{H} \mathrm{I} \mathrm{Ly} \alpha$, is $\Delta z=$ $[2.667,3.751]=1.085$. For $[\mathrm{O}$ II $]$ and $[\mathrm{O}$ III $]$ these values are $\Delta z=$ $[0.196,0.550]=0.354$, and $\Delta z=[0 ., 0.154]=0.154$, respectively. The solid angle subtended by the slit is $0.252 \mathrm{arcmin}^{-2}$.

The number of objects per unit redshift per square arcminute for $\operatorname{Ly} \alpha$ is given by

$$
\frac{\partial^{2} \mathcal{N}}{\partial z \partial \Omega}=3.66 \mathcal{N}_{\mathrm{Ly} \alpha}=98.7
$$

where $\mathcal{N}_{\text {Ly } \alpha}=27$ is the total number of objects. For [O II] this value is

$$
\left.\frac{\partial^{2} \mathcal{N}}{\partial z \partial \Omega}=11.2 \mathcal{N}_{[\mathrm{O}}{ }_{\mathrm{II}}\right]=302.7,
$$

for $27+4=31$ putative [O II $]$ emitters.

For [O III],

$$
\frac{\partial^{2} \mathcal{N}}{\partial z \partial \Omega}=25.77 \mathcal{N}_{[\mathrm{O}}{ }_{\mathrm{II}]}=412.3
$$

with $\mathcal{N}_{[\mathrm{O}}$ III $]=16$ objects in the right wavelength range.

For the case of Ly $\alpha$ the (cumulative) distribution $\partial^{2} \mathcal{N}_{>F_{\text {Ly } \alpha}} /$ $\partial z \partial \Omega$ of those objects exceeding a line flux $F_{\mathrm{Ly} \alpha}\left[\mathrm{erg} \mathrm{s}^{-1} \mathrm{~cm}^{-2}\right]$ is given by Figure 7 .

The comoving survey volume is given by

$$
d V_{c}=\frac{c}{H_{0}}(1+z)^{2} D_{A}^{2}(z) d \Omega \frac{d z}{E(z)},
$$




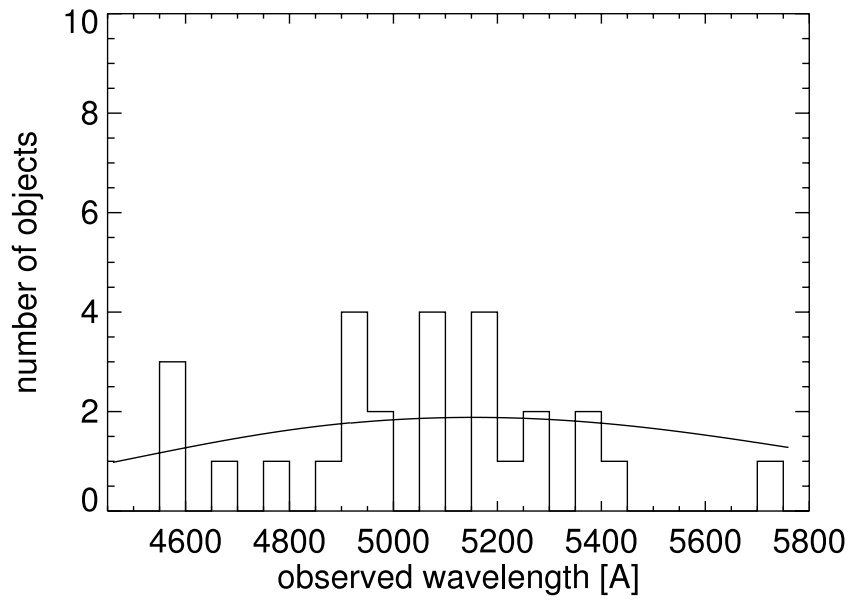

FIG. 5.-Distribution of the emitters in observed wavelength. The solid line is the sensitivity of the instrument, in arbitrary units. The actual detection threshold probably drops faster toward the edges because of illumination, dithering losses, and detector artifacts.

with the angular diameter distance $D_{A}(z)$,

$$
D_{A}(z)=\frac{c}{H_{0}(1+z)} \int_{0}^{z} \frac{d z^{\prime}}{E\left(z^{\prime}\right)},
$$

and

$$
E(z)=\sqrt{\Omega_{m}(1+z)^{3}+\Omega_{\Lambda}} .
$$

Then the comoving volumes represented by the two-dimensional spectrum are $V_{c}=885 \mathrm{Mpc}^{3} h_{70}^{-3}$ for Ly $\alpha, V_{c}=57.5 \mathrm{Mpc}^{3} h_{70}^{-3}$ for [O II], and $V_{c}=1.82 \mathrm{Mpc}^{3} h_{70}^{-3}$ for [O III]. The total comoving number density of objects detected, $d \mathcal{N} / d V_{c}$, is then $0.030,0.53$, and $14.9 \mathrm{Mpc}^{-3} h_{70}^{3}$ for $\mathrm{H} \mathrm{I}$, [O II], and [O III], respectively.

The line luminosity is given by

$$
L=4 \pi D_{L}^{2}(z) F
$$

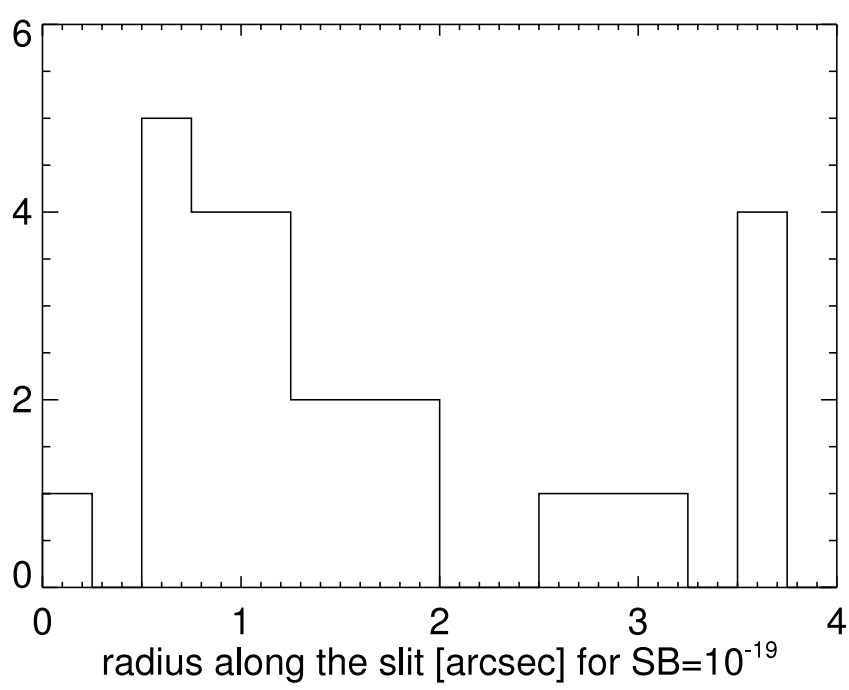

FIG. 6.-Distribution of the projected radius along the slit, i.e., the distance of the $10^{-19}$ surface brightness contour (based on the model profile) from the center of the emitter. The four cases where the contour extends beyond our fitting range are collected in the $3.6^{\prime \prime}$ bin, but the contour may reach considerably larger distances than that.

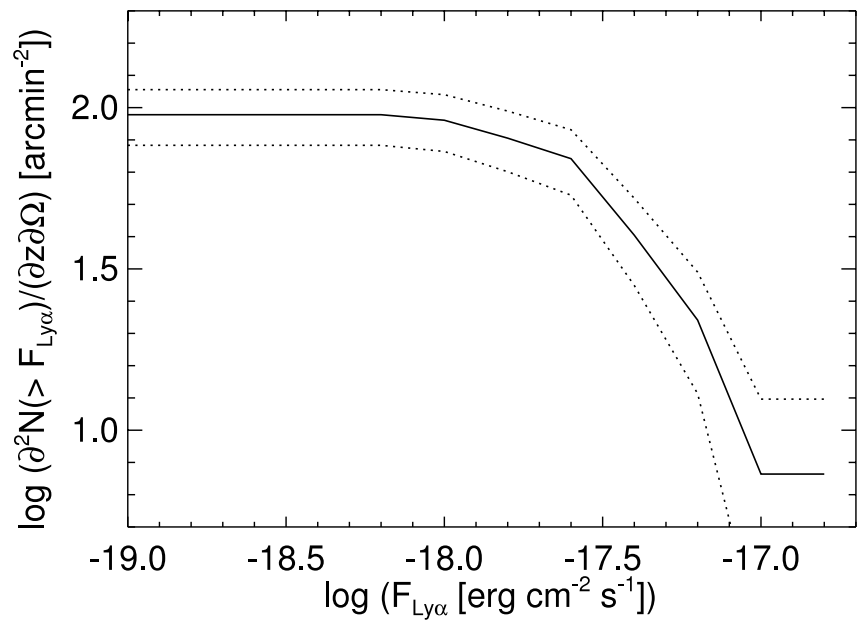

FIG. 7.- Number of emitters per unit redshift and square arcminute with a line flux exceeding $F_{\text {Ly } \alpha}$.

with the line flux $F$ and the luminosity distance $D_{L}=(1+z)^{2} D_{A}$, and $D_{A}$ as given above.

The histogram of luminosities for $\mathrm{H}_{\mathrm{I}} \mathrm{Ly} \alpha$ is given in Figure 8. Figures 9, 10, and 11 show the cumulative comoving density of objects versus luminosity, again under the assumption that the objects are entirely either $\mathrm{H}_{\mathrm{I}} \mathrm{Ly} \alpha$, [O II], or [O III] emitters. All luminosity functions are calculated from the fluxes measured in the larger, $2^{\prime \prime} \times 7.6^{\prime \prime} \times 1510 \mathrm{~km} \mathrm{~s}^{-1}$ aperture, which is related to the fluxes from the smaller $2^{\prime \prime} \times 2^{\prime \prime}$ one (Table 2, col. [4]) by the factors given in column (5).

\subsection{Rate of Incidence per Unit Redshift}

Each population of emitters produces a total "footprint" in the plane of the sky, which can be used to calculate the rate of incidence along a line of sight, e.g., in a QSO spectrum, once the number density and cross section on the sky are known.

The contribution of the emitter population to the rate of incidence per unit redshift, $d N / d z$, is given by

$$
\frac{d N}{d z}=\sum_{i} \frac{\sigma_{i}}{V_{i}} \frac{d l}{d z},
$$

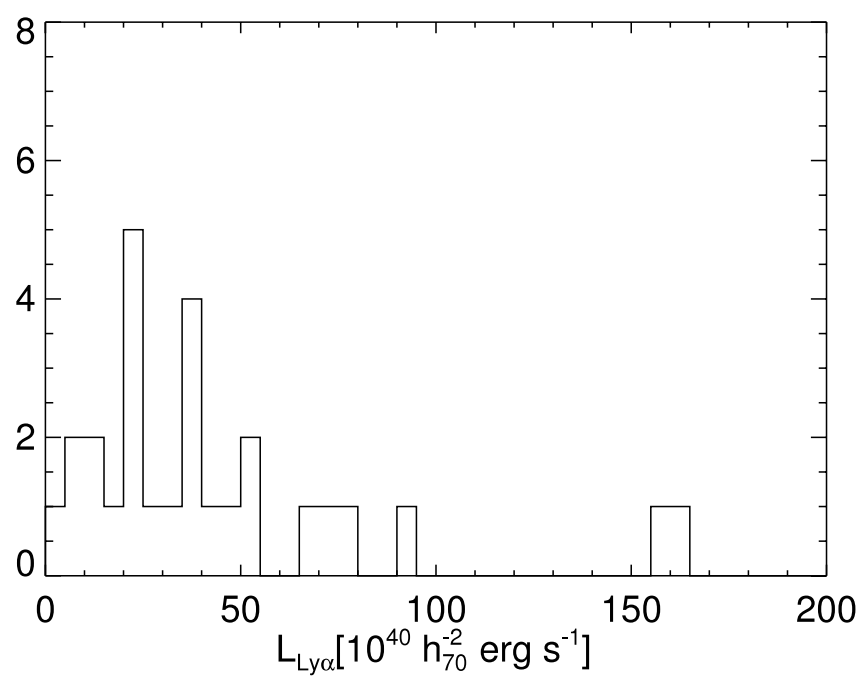

FIG. 8.-Frequency distribution of luminosities $L_{\mathrm{Ly} \alpha}$. 


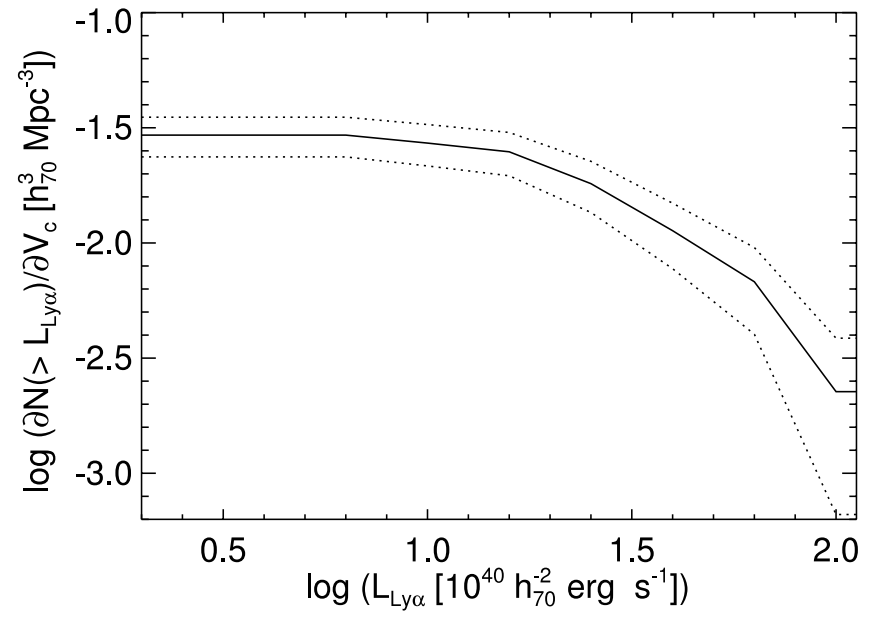

FIG. 9.-Comoving density of emitters with a luminosity exceeding $L_{\mathrm{Ly} \alpha}$.

where the sum is over emitters with index $i, V_{i}$ is the comoving volume in which object $i$ could have been detected, $\sigma_{i}$ the comoving spatial cross section of emitter $i$, and the comoving distance per unit redshift at redshift $z_{i}$ is

$$
\frac{d l\left(z_{i}\right)}{d z}=\frac{c}{H_{0} E\left(z_{i}\right)} .
$$

We use the distribution of sizes (Fig. 6) to compute the comoving spatial cross sections $\sigma_{i}$ and plot the resulting cumulative $d N / d z$ as a function of object size in Figure 12, where each graph is derived as if all objects where either entirely $\mathrm{H}_{\mathrm{I}} \mathrm{Ly} \alpha$, [O II] 3727 , or [O III] 5007 emitters. The short vertical lines denote the resolution limit, telling us that objects with nominal sizes smaller than that may not be contributing as much to the cross section on the sky and thus to the $d N / d z$. This correction is relatively small for all cases.

\section{THE IDENTITY OF THE EMITTERS}

Most of our objects are technically single line objects, too close to the detection threshold to study the precise line shape or detect other weaker lines that should also be present (as in the case of [O III]). Moreover, for most of them neither the spectrum nor a deep Keck LRIS $V$-band image (see below) show significant continua.

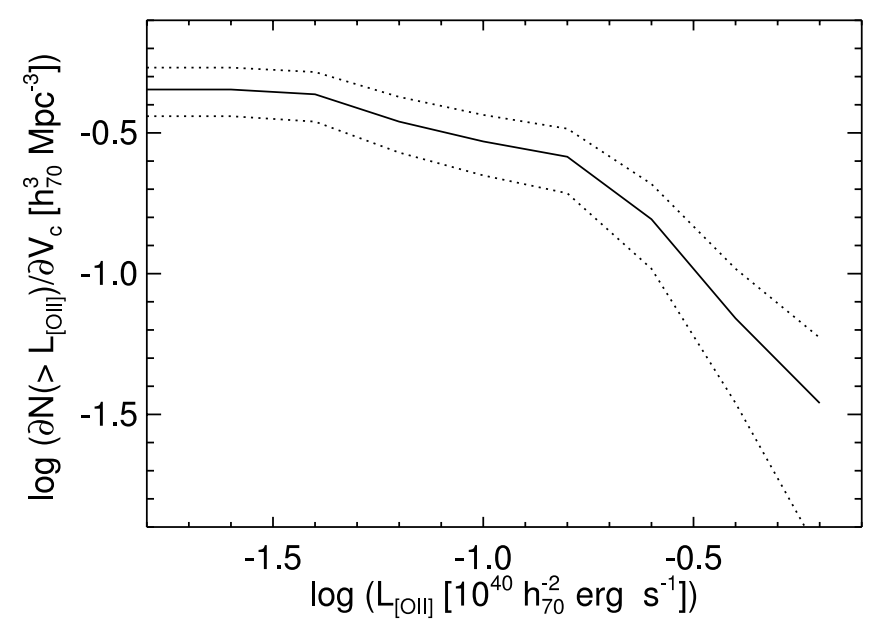

FIG. 10.-Comoving density of emitters under the assumption that they are [O II] $3728 \AA$, with a luminosity exceeding $L_{[\mathrm{O} \text { II] }}$.

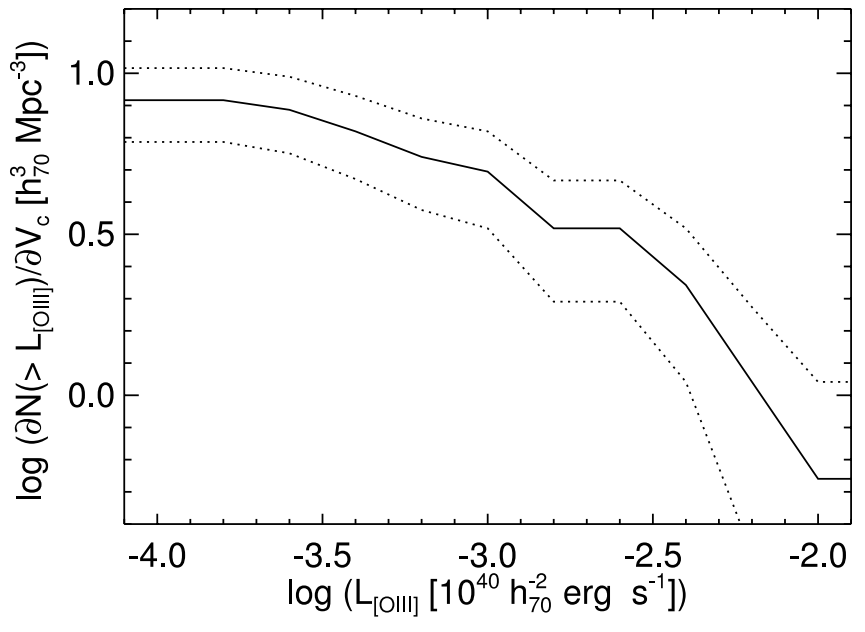

Fig. 11.-Comoving density of emitters under the assumption that they are O III $5007 \AA$, with a luminosity exceeding $L_{[\mathrm{O} \text { I] }}$.

Judging from the emission-line profiles alone, we estimate conservatively that at least $\sim 6$ objects are likely to be high-redshift $\operatorname{Ly} \alpha$, because of their pronounced asymmetric emission profiles. A further three objects seem to coincide closely with QSO Ly $\alpha$ forest absorption systems (see below), which makes them relatively secure $\mathrm{H}$ i identifications.

Thus, from the spectroscopic evidence discussed so far it is not possible to exclude the possibility that the majority of our objects are low-redshift contaminants from [O II] or [O III]/Balmer series emitters. We shall now present a number of arguments that will help us to judge the plausibility of these alternatives.

\subsection{Are the Emitters Dominated by [O II] at Low Redshift?}

In addition to the four objects already eliminated based on their bright [O II] doublets and continua (see $\S 3$ ), there is a similarly small number among the 27 remaining objects whose line profiles seem to be at least consistent with [O II]. We can only barely resolve the [O II] 3726, 3728 doublet in our spectra, but we estimate that we see up to four objects with potential multiple emission peaks (probably including noise spikes) at the right wavelength separation, which are at least consistent with [O II] (although none of them has to be). Given our rather poor spatial resolution of

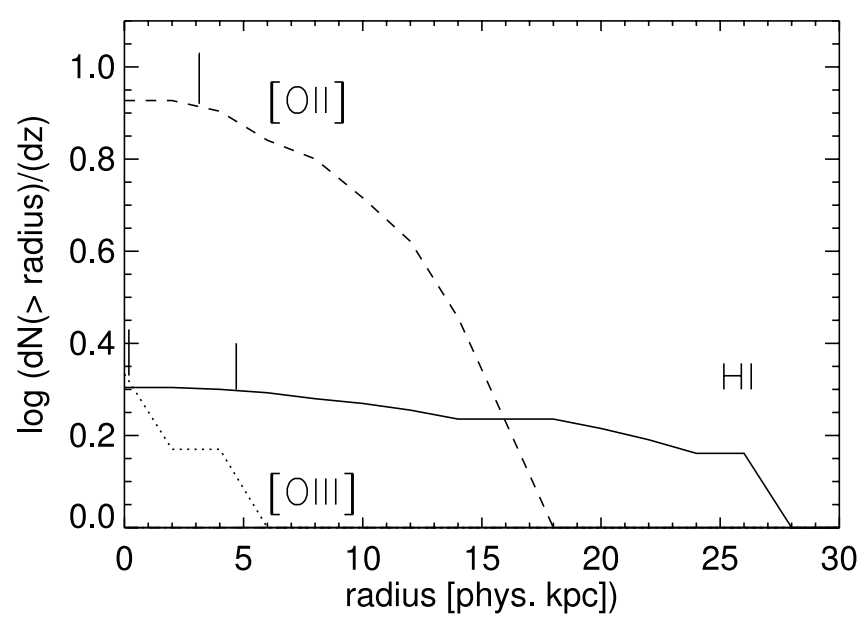

FIG. 12.-Contribution of objects of different sizes to the rate of incidence per unit redshift, $d N / d z$, for $\mathrm{H}$ I (solid line), [O II] (dashed line), and [O III] (dotted line). The short vertical lines riding on top of the curves indicate the spatial resolution limit along the slit. 
4-8 kpc at these redshifts, we should probably expect any additional [O II] emitters to reside among our spatially unresolved sources.

If our emitters were [O II], the luminosities would range from $3.7 \times 10^{38}$ to $2 \times 10^{40} \mathrm{erg} \mathrm{s}^{-1}$, and the redshifts from $z=0.196$ to 0.550 . Using a standard calibration for the relation between star formation rate and [O II] luminosity (Kennicutt 1998),

$$
\operatorname{SFR}\left(M_{\odot} \mathrm{yr}^{-1}\right)=1.4 \times 10^{-41} L_{[\mathrm{O} \text { II }]}\left(\mathrm{erg} \mathrm{s}^{-1}\right),
$$

these luminosities correspond to star formation rates between $5 \times 10^{-3}$ and $0.3 M_{\odot} \mathrm{yr}^{-1}$. The total inferred [O II] luminosity density of $1.0 \times 10^{41} \mathrm{erg} \mathrm{s}^{-1} \mathrm{Mpc}^{-3}$ would correspond to a star formation rate density of $1.42 M_{\odot} \mathrm{yr}^{-1} \mathrm{Mpc}^{-3}$.

The space density corresponds to $0.53(N / 31) \mathrm{Mpc}^{-3}$. We can estimate the number of expected [O II] detections from the field galaxy luminosity function of Trentham et al. (2005). We are able to detect emitters with line fluxes $>10^{-18} \mathrm{erg} \mathrm{cm}^{-2} \mathrm{~s}^{-1}$. Most [O II] emitters (Hogg et al. 1998) do not exceed a rest equivalent width of $50 \AA$. If we use this value to convert our line flux detection threshold into continuum magnitudes, and we adopt the redshift 0.364 , which divides the volume where we can detect [O II] into a lower and higher redshift half, we find $M=-12.8$ as the faintest continuum magnitude where we would be able to detect the corresponding [O II] emission. The total space density of local field dwarf galaxies down to an absolute magnitude of $M_{R}=-13$ is about $8 \times 10^{-2} \mathrm{Mpc}^{-3}$ (Trentham et al. 2005). Based on that estimate, about five of our emitters are indeed likely to be due to [O II] emission in low-redshift dwarf galaxies. This number fits in well with the four [O II] emission-line galaxies which we have already identified, outside of the 27 faint emitters. Just from Poissonian arguments (again assuming that we are not looking at a cluster), the probability that there are 2 more additional [O II] emitters in our volume is about $22 \%$; the probability that there are more than 4 more is only about $7 \%$.

The rate of incidence of low-redshift damped Ly $\alpha$ systems is another (albeit somewhat uncertain) indication that our emitters are not likely to be predominantly [O II]. If we assume that all starforming galaxies are embedded in DLAs (are a subset of DLAs), and that the radius of optically thick gas is very likely to be larger than that of [O II] emission, the product of the number density and cross section of [O II] emitters cannot be larger than that of DLAs. Our inferred total $d N / d z$ (Fig. 12) for [O II] at 0.93 is more than 14 times larger than that of its contemporary damped Ly $\alpha$ systems, as estimated from the Sloan Digital Sky Survey, $d N / d z(\bar{z}=$ $0.37)=0.066$ (Rao et al. 2006), so even if DLAS were not larger than [O II] emission regions, and not more numerous, only about $7 \%$ of our emitters (i.e., two objects in total, or none in the remaining sample of 27) should be [O II] to not violate the $d N / d z$ constraint from DLAS.

\subsubsection{Are the Emitters Dominated by O III at Even Lower Redshift?}

As for [O III $] 5007 \AA$, there is a total of 16 emitters in the redder $769 \AA$ long part of the spectrum where [O III] could be detected. The remaining 12 emitters occupy the bluer $550 \AA$, where the wavelength is below the rest-frame wavelength of [O III] and obviously cannot be [O III]. The ratio between the numbers of objects per wavelength in the [O III] region and those in the non-[O III] region is then $0.95 \pm 0.23$, i.e., there is no significant enhancement of the line density, making a dominant contribution from [O III] emitters unlikely (a similar line of reasoning can be employed against the emitters being [O III] $4959 \AA$, etc.).

The corresponding luminosities would range from $2.0 \times 10^{36}$ to $3.2 \times 10^{38} \mathrm{erg} \mathrm{s}^{-1}$ at redshifts between $z=0$ and 0.154 . The space density corresponds to $9(N / 16) \mathrm{Mpc}^{-3}$, about 40 times higher than the local space density of dwarf galaxies down to an absolute magnitude of $M_{R}=-9\left(0.23 \mathrm{Mpc}^{-3}\right.$; Trentham et al. 2005). As we have already found one galaxy in the right redshift range outside of our emitter sample (although it had only $\mathrm{H} \beta$ and no actual [O III] emission; see $\S 2$ ), the Poissonian probability to have one or more additional ones hidden in our sample is less than $7 \%$; the probability to have two or more is less than $1 \%$. There is no obvious foreground cluster in our field, and it seems thus very unlikely that even one of these emitters is due to [O III] emission from an $\mathrm{H}$ II region in a low-redshift dwarf galaxy. Note, however, that curiously, the lower end of the inferred luminosities at the smallest distances corresponds to that of bright planetary nebulae (PNe; 4 objects). Gerhard et al. $(2005,2007)$ have searched for PNe in the core of the Coma Cluster with a multiple slitlet technique to similar limiting fluxes. They found $35 \mathrm{PNe}$ candidates in a similarly sized volume, but centered on the core of the Coma Cluster, where the overdensity of PNe should be very large. It thus appears unlikely that in a random field we should have found [O III] emission from bright PNe. The rate of incidence of DLAs (at mean redshift 0.08) constrains the fraction of [O III] emitters, too, limiting it to be less than $15 \%$ of our emitters.

We conclude that we are likely to have found (and eliminated already) most of the [O II] contaminants in our emitter sample, as predicted by the space density of the local galaxy population, and the rate of incidence of low-redshift damped Ly $\alpha$ systems. It is even less likely that there are [O III] $5007 \AA$ contaminants in our sample. Therefore, from here on we shall treat the remaining emitters as $\mathrm{H}_{\mathrm{I}} \mathrm{Ly} \alpha$ and discuss the implications, keeping in mind that some of the objects may still be misidentified.

\section{Ly $\alpha$ EMITTERS AT REDSHIFTS $2.666<z<3.751$}

If our objects are Ly $\alpha$ emitters, the observed fluxes correspond to luminosities between $7.9 \times 10^{40}$ and $1.6 \times 10^{42} \mathrm{erg} \mathrm{s}^{-1}$. If caused by star formation, the range of luminosities corresponds to star formation rates of $7 \times 10^{-2}$ to $1.5 M_{\odot} \mathrm{yr}^{-1}$, where we have used the standard relation

$$
\operatorname{SFR}\left(M_{\odot} \mathrm{yr}^{-1}\right)=9.1 \times 10^{-43} L_{\mathrm{Ly} \alpha}\left(\mathrm{erg} \mathrm{s}^{-1}\right)
$$

for the Ly $\alpha$ luminosity as a function of star formation rate (based on Kennicutt 1998 and case B assumptions for the conversion of $\mathrm{H} \alpha$ and Ly $\alpha$; Brocklehurst 1971). Note again that the actual values could be larger by a factor of a few due to slit losses. The total Ly $\alpha$ luminosity density of $1.4 \times 10^{40} \mathrm{erg} \mathrm{s}^{-1} \mathrm{Mpc}^{-3}$ corresponds to a star formation rate density of $1.2 \times 10^{-2} M_{\odot} \mathrm{yr}^{-1}$ $\mathrm{Mpc}^{-3}$, about $36 \%$ of the value (uncorrected for dust) inferred for $B$-band dropouts in the Hubble Ultra Deep Field by Bouwens et al. (2007).

The inferred space density is $3.0(N / 27) 10^{-2} \mathrm{Mpc}^{-3}$, a factor of 3 smaller than the total space density of local dwarf galaxies, but an order of magnitude larger than the space density of previously known Ly $\alpha$ emitters at this redshift (but with our study going down to much lower flux limits). If our detected emissionline objects are primarily due to $\operatorname{Ly} \alpha$, this corresponds to a significant steepening of the luminosity function of Ly $\alpha$ emitters at luminosities below $\sim 10^{42} \mathrm{erg} \mathrm{s}^{-1}$.

Is such a numerous population of $\operatorname{Ly} \alpha$ emitters plausible? The inferred space density is similar to the space density of $B$ dropouts in the HUDF at slightly larger redshifts (Bouwens et al. 2007; see also below, and Fig. 15), and in fact, less by factors of 10-30 than the number density inferred by Stark et al. (2007) for $z \sim 8-$ 10 objects. Intriguingly, the above-mentioned survey for planetary 


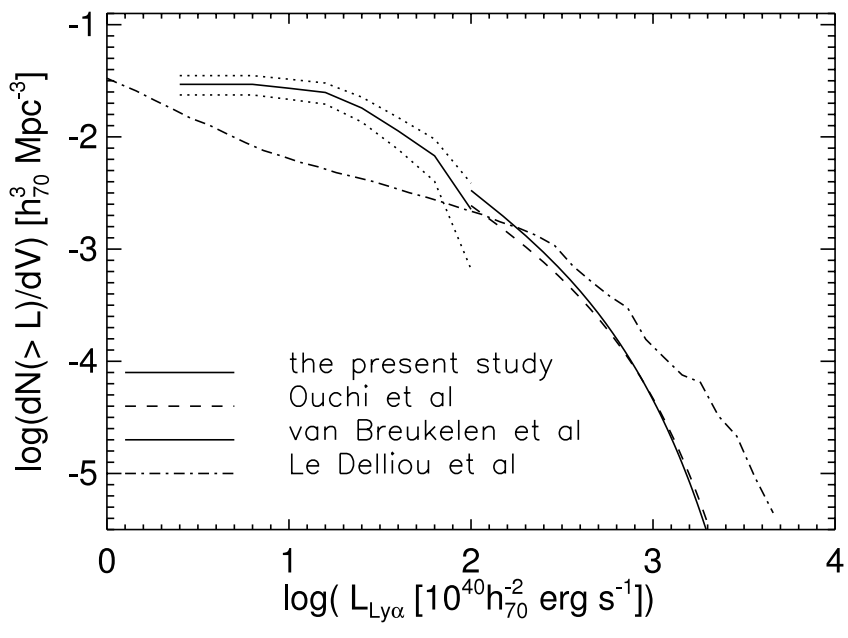

FIG. 13.-Observed cumulative luminosity function at $z=3.1$ from Ouchi et al. (2008; dashed line), $z=2.9$ from van Breukelen et al. (2005; doubledot-dashed), predictions from Le Delliou et al. (2005, 2006; dash-dotted line). and our sample (solid line, with the dotted lines representing the $1 \sigma$ error contours). There is almost continuity in amplitude and slope in the overlap region with the brighter observational data. The van Breukelen et al. function with its adopted $\alpha=-1.6$ slope, if continued beyond its measured range, is never more than 0.2 dex below our curve. The theoretical curve from LeDelliou et al. for a constant escape fraction is shallower than all the observed distributions at all luminosities, but the gap between it and our function steepens toward fainter luminosities. Our observed distribution starts to flatten near $3 \times 10^{41} \mathrm{erg} \mathrm{s}^{-1}$, well above the detection threshold, so the turnover may be intrinsic.

nebulae by Gerhard et al. (2005) also found 20 " background objects" that could not be identified otherwise, in a similar volume. Other dedicated surveys for $\operatorname{Ly} \alpha$ emitters at $z \sim 2.5-3.5$ (e.g., Hu et al. 1998; Kudritzki et al. 2000; Steidel et al. 2000; Stiavelli et al. 2001; Fujita et al. 2003; van Breukelen et al. 2005; Gronwall et al. 2007; Ouchi et al. 2008) appear consistent with our survey (there is little overlap in the range of fluxes reached). Our objects have about 20 times the volume density of, for example, the objects found by the shallower Gronwall et al. survey (their detection threshold is $1.5 \times 10^{-17} \mathrm{erg} \mathrm{cm}^{-2} \mathrm{~s}^{-1}$ ).

Figure 13 shows a comparison of our cumulative luminosity function (solid line, with $1 \sigma$ errors indicated by the dotted lines) with the best fit, $z=2.9$ luminosity function from the IFU survey by van Breukelen (dash-triple-dotted line), the $z=3.1$ (narrowband filter) luminosity function of Ouchi et al. (2008), and the predictions by Le Delliou et al. (2006; dash-dotted line). Note again that our luminosity function does not include corrections for slit losses. At the bright end of our sample (near $\sim 10^{42} \mathrm{erg} \mathrm{s}^{-1}$ ) there is good agreement with the two observed luminosity functions, and there is initial agreement in the slope as well in the small region of overlap, but our sample becomes steeper going toward fainter magnitudes. The solid line appears to flatten again toward luminosities below $2 \times 10^{41}$ (or a flux $3 \times 10^{-18}$ in the usual units). Objects at half that flux are still clearly detectable for sources with characteristics similar to ours. This suggests that the flattening may be real, and the numbers may start to decline. One possibility is that we may already be seeing the bulk of the currently star forming galaxies.

From a theoretical point of view, the CDM picture of structure formation predicts a rather steeply rising mass function at low masses, but note that even for a linear light-to-total-halomass relation, the luminosity function required to explain our inferred space density requires an even steeper faint-end slope. Near $10^{42} \mathrm{erg} \mathrm{s}^{-1}$, our observed density of objects is in agreement with the CDM-based model population of Ly $\alpha$ emitters from Le Delliou et al. (2005, 2006; dash-dotted line in Fig. 13), but then it steepens over the next decade in luminosity considerably, relative to the models based on a constant Ly $\alpha$ escape fraction, perhaps suggesting that this fraction may not be constant after all. The discrepancy approaches about a factor of 5 at $2 \times 10^{41} \mathrm{erg} \mathrm{s}^{-1}$ and then decreases again toward fainter magnitudes. Such a steepening of the luminosity function could perhaps be explained if dust extinction becomes increasingly less important for fainter emitters.

Unfortunately, the stellar and total masses of the emitters are very uncertain. If the Ly $\alpha$ emission is due to star formation at the rates estimated above, the accumulated stellar mass within $10^{9} \mathrm{yr}$ is in the range $7 \times 10^{7}$ to $1.5 \times 10^{9} M_{\odot}$. Another estimate of the mass can be obtained by comparing our inferred space density with that of dark matter halos predicted by CDM models. The space density inferred by our sample of objects corresponds to the cumulative space density of dark matter halos with total mass $>3 \times 10^{10} M_{\odot}$ and circular velocities $v_{c}>50 \mathrm{~km} \mathrm{~s}^{-1}$ (e.g., Mo \& White 2002; Wang et al. 2007).

In the next section we examine the competing Ly $\alpha$ production mechanisms, before returning to a discussion of the nature of the emitters in the larger scheme of galaxy populations.

\section{ASTROPHYSICAL ORIGINS OF THE Ly $\alpha$ EMISSION}

At the faint detection threshold attained here, a number of different physical processes can produce Ly $\alpha$ emission at comparable fluxes, and it is not certain that we are necessarily seeing the results of star formation. The faintest of these competing mechanisms is Ly $\alpha$ fluorescence, induced by the general UV background. However, our fluxes (see Table 2) typically exceed the predicted surface brightness limit for individual objects (Gould $\&$ Weinberg 1996) by an order of magnitude. A second source of Ly $\alpha$ photons arises from the presence of a QSO in our field. The QSO locally enhances the UV flux and can in principle boost the surface brightness of $\mathrm{H}$ I to much higher levels, where it can be readily detected. A third effect expected to rear its head at our sensitivity threshold is cooling radiation; gas falls into a galactic potential well and sheds part of its potential energy in the form of Ly $\alpha$ line radiation. These processes are observationally distinct from star formation, in that only star formation actually produces a significant (stellar) continuum as well, which can serve as a discriminant among the various sources of Ly $\alpha$. This question will be addressed briefly in the next section, followed by an investigation of the role of the QSO's local radiation field, and of cooling radiation.

\subsection{Stellar Continuum Emission from the Line Emitter Sample}

To check for continuum emission, we could avail ourselves of $V$-band images taken with the LRIS instrument on the Keck I telescope, with a total exposure time of $5610 \mathrm{~s}$. The combined image was flux-calibrated with the photometric data from Hall et al. (1996). The slit coordinate system was mapped onto the two-dimensional image, and the $V$-band fluxes were measured in appropriately positioned apertures of size $2^{\prime \prime} \times 2^{\prime \prime}$. These apertures are expected to have a typical spatial uncertainty on the order of half a slit width perpendicular to the slit, as the spectrum allows us only to derive the coordinate along the slit.

The $1 \sigma$ detection threshold in this aperture is $3 \times 10^{27} h_{70}^{-2} \mathrm{erg} \mathrm{s}^{-1}$ $\mathrm{Hz}^{-1}$. Figure 14 shows the detected $V$ band luminosities (crosses with error bars), together with $3 \sigma$ upper limits for undetected objects (arrows), as a function of the Ly $\alpha$ luminosity. Objects 1 , 2, 3, and 39 fell off the edges of the $V$-band image and where not constrained. However, object 39 has a detectable continuum in 


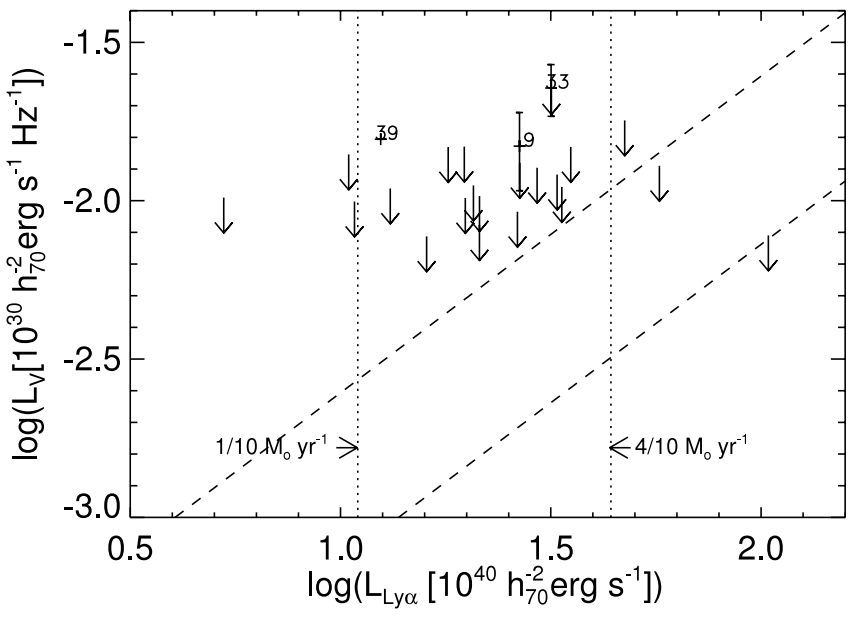

FIG. 14.- $V$-band luminosity density vs. Ly $\alpha$ line luminosities, under the assumption that all sources are high-redshift Ly $\alpha$. The arrows are upper limits, i.e., detected line emitters without $V$-band counterparts at a $3 \sigma$ significance level (in a $2^{\prime \prime} \times 2^{\prime \prime}$ aperture). Of the three positive detections 39,9 , and 33 , the latter is somewhat off center and may be a low- $z$ source or a chance coincidence of a high-redshift emitter with an lower redshift continuum source. Three objects, 1 , 3 and 39, are not covered by the $V$-band image. The spectrum of 39 , however, shows a continuum consistent with the expected Ly $\alpha$ forest decrement. We have given here its $1500 \AA$ rest-frame luminosity measured from the spectrum instead of the $V$-band luminosity. The lower diagonal dashed line is the expected locus of Ly $\alpha$ emitters assuming that both, UV luminosity and Ly $\alpha$ flux, were produced by star formation only (see text). This line corresponds to a rest-frame equivalent width of $68 \AA$ and would intercept the $y$-axis at -4.14 . The higher dashed line with the same slope delineates $\mathrm{EW}=20 \AA$. The vertical dotted lines in Ly $\alpha$ emitters indicate star formation rates of 1/10 (left) and 4/10 (right) $M_{\odot} \mathrm{yr}^{-1}$.

the spectrum itself, and shows a clear Ly $\alpha$ forest decrement. Its data point in the plot gives the $1500 \AA$ continuum flux measured directly from the spectrum.

As for the other objects, visual inspection shows that very few objects selected by the presence of Ly $\alpha$ emission in the spectrum show up in the $V$-band image. At the $3 \sigma$ flux level, only two objects have automatically detectable $V$-band counterparts, namely 9 and 33, both of which could be low-redshift continuum sources or high-redshift line emitters experiencing chance coincidences with lower redshift continuum sources. Interestingly, these are the same two objects picked out by eye as having clear continuum counterparts. In the spectrum itself, several objects coincide with apparent continuum traces (all very faint), many of which, with the exception of the above-mentioned object 39 , are consistent with bad rows or charge-transfer problems, or accidental spatial coincidence with unrelated continuum sources.

It is instructive (and sobering) to consider where in the continuum-line luminosity diagram (Fig. 14) star-forming galaxies should reside, were we able to detect them in both continuum and line emission. Adopting again equation (12) for the Ly $\alpha$ luminosity as a function of star formation rate, and

$$
L_{\mathrm{UV}}\left(\mathrm{erg} \mathrm{s}^{-1} \mathrm{~Hz}^{-1}\right)=8 \times 10^{27} \operatorname{SFR}\left(M_{\odot} \mathrm{yr}^{-1}\right)
$$

for the UV continuum luminosity (for a Salpeter IMF and solar metallicity; Madau et al. 1998), we equate the star formation rates in these relations to obtain the dashed line in the bottom right corner of Figure 14. This delineates the positions of galaxies where both UV continuum flux and Ly $\alpha$ line flux are entirely due to star formation, and is given by

$$
\log \left(L_{\mathrm{UV}}\right)=-14.14+\log \left(L_{\mathrm{Ly} \alpha}\right)
$$

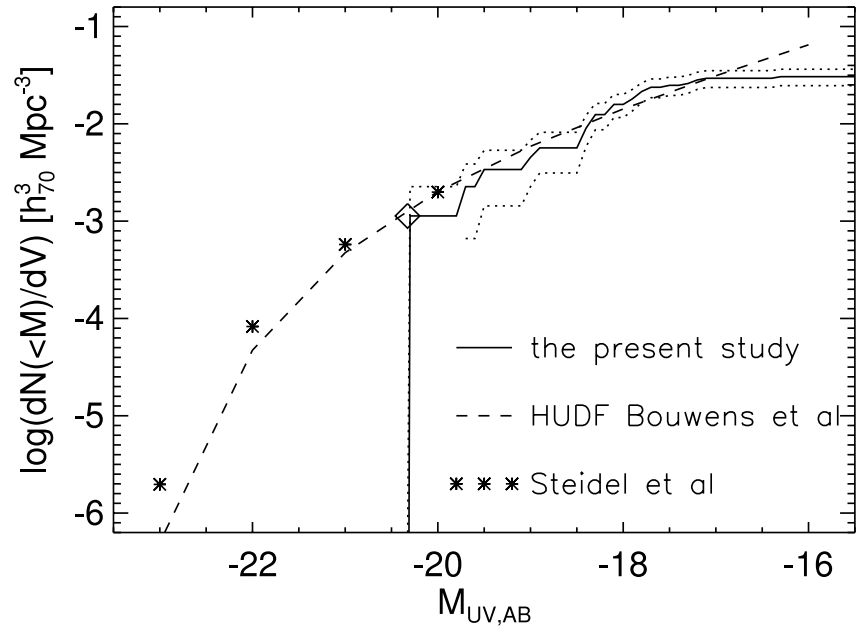

FIG. 15.-Cumulative UV continuum luminosity functions of Steidel et al. (1999; asterisks), the Hubble Ultra Deep Field (Bouwens et al. 2007; dashed line), and the cumulative distribution of our survey (solid line; dotted lines are $\pm 1 \sigma$ errors). The diamond symbol shows object 39 . The emitters are entered with a continuum magnitude predicted by their Ly $\alpha$ line flux, as we have only upper limits on the continuum (see text). The number density of our emitters closely corresponds to the number density of Bouwens et al. (2007). The absence of objects brighter than -21 is consistent with our small survey volume.

The Ly $\alpha$ rest-frame equivalent width formally implied in equation (14) is $68 \AA$, a value high for color-selected galaxies (Shapley et al. 2003) but not exceptional even for much brighter Ly $\alpha$ emitters (e.g., Gronwall et al. 2007). There is also an upper diagonal dashed line, showing the locus for a rest equivalent width of $20 \AA$. The dotted vertical line to the left gives the Ly $\alpha$ flux for a star formation rate of $1 / 10 M_{\odot} \mathrm{yr}^{-1}$, the one to the right for $4 / 10 M_{\odot} \mathrm{yr}^{-1}$.

Unfortunately, most of our objects are predicted to be too faint in the continuum to be able to test whether star formation is the origin of the $\operatorname{Ly} \alpha$, so the continuum flux measurement or, equivalently, the equivalent widths are not helpful here. The nondetection of the continuum is of course fully consistent with star-formationinduced Ly $\alpha$ emission.

The position of object 39 so far from (to the left of) the SF locus suggests that the Ly $\alpha$ emission is heavily suppressed, e.g., by dust, as seems to be the case for massively star-forming galaxies (e.g., Shapley et al. 2003).

The situation is summarized in Figure 15, where we compare the cumulative UV continuum luminosity functions of Steidel et al. (1999; asterisks) and the Hubble Ultra Deep Field $z \sim 4$ $B$-band dropouts (Bouwens et al. 2007; dashed line) with the line emitters in our survey (solid line). For all but object 39 (where we have an actual measurement), we have assigned "continuum magnitudes" based on equation (14). Note that this is just a scheme to show the predicted continuum if both UV continuum and Ly $\alpha$ line radiation were entirely due to star formation, ignoring any extinction effects.

Given our small survey volume, only about one object in our entire survey should be bright enough to show up in a groundbased, broadband-color survey, i.e., as a "Lyman break" galaxy (Steidel et al. 1999), and this is what we find (namely, number 39). Object 39 brings the number of galaxies brighter than $-20.3 \mathrm{AB}$ mag up to unity, virtually identical to the prediction from the integrated continuum luminosity functions for a volume of our size. Our volume is too small to have a much brighter galaxy in it. The total number density of our emitters is comparable to the number density of the Bouwens et al. study at magnitudes brighter than $M_{\mathrm{AB}}=-16.5$. The "luminosity function" for the 
line emitters appears to be steepening between -20 and -18 , a behavior already seen above in our comparison with the Ly $\alpha$ emitters. It could be indicative of dust extinction decreasing toward fainter magnitudes. A correction for dust would reduce the slope of the line-emitter luminosity function, bringing it into better agreement with the Bouwens et al. curve. We caution, however, that our objects cannot be strictly identical to the class of $B$-band dropouts, as half of them are at lower redshift.

\subsection{Lya Fluorescence Induced by the QSO?}

We turn next to the possibility that the Ly $\alpha$ radiation arises from patches of optically thick hydrogen gas, induced to Ly $\alpha$ fluorescence by the ionizing radiation from the QSO in our field. The basic idea is that the partial conversion of the QSO UV radiation field into Ly $\alpha$ photons at the surface of optically thick hydrogen bodies raises the emission from clouds or galaxies in the QSO vicinity above the detection level. This effect has been studied by a number of authors (Fynbo et al. 1999; Francis \& Bland-Hawthorn 2004; Francis \& McDonnell 2006; Adelberger et al. 2006; Cantalupo et al. 2007). The spatial extent of the zone of influence is obviously inversely proportional to the square root of the intensity of the fluorescent emission. Following Cantalupo et al. (2005), we can express the enhanced Ly $\alpha$ flux in terms of a boost factor, i.e., the ratio of the surface brightness enhanced by the QSO $(S)$ to the one caused by the general UV background $\left(S_{\mathrm{bg}}\right)$,

$$
\frac{S}{S_{\mathrm{bg}}}=\left(0.74+0.5 b^{0.89}\right),
$$

where

$$
b=15.2 \frac{L_{\mathrm{LL}}}{10^{30} \mathrm{erg} \mathrm{s}^{-1} \mathrm{~Hz}^{-1}} \frac{0.7}{\alpha}\left(\frac{r}{\text { phys. Mpc }}\right)^{-2} .
$$

Both the luminosity of the QSO at the Lyman limit, $L_{\mathrm{LL}}$, and its precise systemic redshift are critically important ingredients in this calculation. Estimating the latter from the position of the $\mathrm{O}_{\mathrm{I}}$ $\lambda 1302 \AA$ emission line, we determine the QSO systemic redshift as $z_{\mathrm{em}}=3.32209$. The luminosity per unit wavelength is given by

$$
L\left(\lambda /\left(1+z_{\mathrm{em}}\right)\right)=4 \pi\left(1+z_{\mathrm{em}}\right) D_{L}^{2}(z) f(\lambda) .
$$

With $f(1040 \AA)=2.2 \times 10^{-17} \mathrm{erg} \mathrm{cm}^{-2} \mathrm{~s}^{-1} \AA^{-1}$ measured directly from our fluxed QSO spectrum, we arrive at a luminosity per unit wavelength $L(1050 \AA)=9.405 \times 10^{42} \mathrm{erg} \mathrm{s}^{-1} \AA^{-1}$. To measure the number of $\mathrm{H}$ I ionizing photons, we still need to determine the luminosity at the Lyman limit and the power-law dependence for wavelengths below the ionization threshold. According to the study by Scott et al. (2004), the power-law index for a QSO with $\log \lambda L(1050 \AA) \approx 9.8 \times 10^{45} \mathrm{erg} \mathrm{s}^{-1}$, where

$$
L(\nu)=L\left(\nu_{0}\right)\left(\nu / \nu_{0}\right)^{\alpha}
$$

is (statistically) consistent with $\alpha \sim-1.5$ (in good agreement with $\alpha \sim-1.57$ for the radio-quiet sample from Telfer et al. 2002).

Extrapolating the luminosity from $1050 \AA$ to the Lyman limit with

$$
L(\lambda)=\frac{c}{\lambda_{0}^{2}} L\left(\nu_{0}\right)\left(\frac{\lambda_{0}}{\lambda}\right)^{\alpha+2}
$$

we get

$$
L(\nu)=2.798 \times 10^{30}\left(\frac{\nu}{\nu_{\mathrm{LL}}}\right)^{-1.5} \mathrm{erg} \mathrm{s}^{-1} \mathrm{~Hz}^{-1}
$$

Inserting these results in the above relation for the boost factor gives

$$
r=\frac{3.018}{\left(S / S_{\mathrm{bg}}-0.75\right)^{0.5618}} \text { phys. Mpc. }
$$

For the relatively faint QSO DMS 2139.0-0405, to boost the surface flux from the background value $S_{\mathrm{bg}}=3.67 \times 10^{-20} \mathrm{erg} \mathrm{cm}^{-2}$ $\mathrm{s}^{-1} \operatorname{arcsec}^{-2}$ to a typical surface brightness of $S \geq 10^{-18} \mathrm{erg} \mathrm{cm}^{-2}$ $\mathrm{s}^{-1} \operatorname{arcsec}^{-2}$ as observed would require the object to be within only 0.479 proper Mpc or 2.07 comoving Mpc. This distance corresponds radially to 254.8 spatial pixels along the slit (about $1 / 4$ of the length of the field), but only 4.3 (!) pixels in the dispersion direction. Figure 16 shows the highly eccentric elliptical contour within which to expect the enhancement to $10^{-18} \mathrm{erg} \mathrm{cm}^{-2}$ $\mathrm{s}^{-1} \operatorname{arcsec}^{-2}$. Only one object, ID 16, falls within the ellipse, and with its maximum surface brightness of $\sim 10^{-18}$ and absence of a continuum is consistent with fluorescing in the ionizing field of the QSO. The overwhelming majority of our sources, however, appear to be oblivious to the QSO's proximity.

\subsection{Signs of Radiative Transfer, and Cooling Radiation}

The spectral line shapes and sizes of our emitters suggest that the Ly $\alpha$ photons may have been processed by radiative transfer through an optically thick $\mathrm{H}$ I medium. The trapping by and protracted escape of line radiation from such a medium should lead to random walk in the spatial and frequency domain. The result may be observable as emission broadened in frequency space and extended in the spatial direction beyond the extent of the actual source of Ly $\alpha$ photons (e.g., Adams 1972; Neufeld 1990; Zheng \& Miralda-Escudé 2002; Dijkstra et al. 2006a; Tasitsiomi 2006). The data appear to show some evidence for these mechanisms at work. The large velocity widths (see Table 2) and radial extent (median projected radius along the slit $7.7 \mathrm{kpc}$ proper, and considerably larger in individual cases) that we have observed are thus suggestive of the signatures of radiative transfer. The FWHM velocity widths measured from optimally extracted spectra of the individual emission-line regions are plotted in Figure 17 versus the power-law index of the surface brightness model (eq. [1]). In some cases these widths are underestimates, because only a single peak was fitted, as opposed to a doublehumped or more complex structure. In that plot, the area to the left of the spectral resolution, about $286 \mathrm{~km} \mathrm{~s}^{-1}$ FWHM, is visible as a zone of avoidance, and between one-third and half of the measured velocity widths clearly exceed the resolution. Sources with large velocity widths seem to prefer smaller power-law indices, i.e., spatial surface brightness profile that drop less rapidly with radius.

\subsubsection{Spatial Surface Brightness Profiles and Fluxes}

To learn more about the topography of the Ly $\alpha$ source and the origin of the radiation, we can attempt to compare our average measurements of the sizes, peak surface brightness, and total fluxes to the models by Dijkstra et al. (2006). With the number of free parameters and the simplifications in these models and the observational complication of the long-slit technique, it is difficult to make a quantitative comparison, but we can at least 


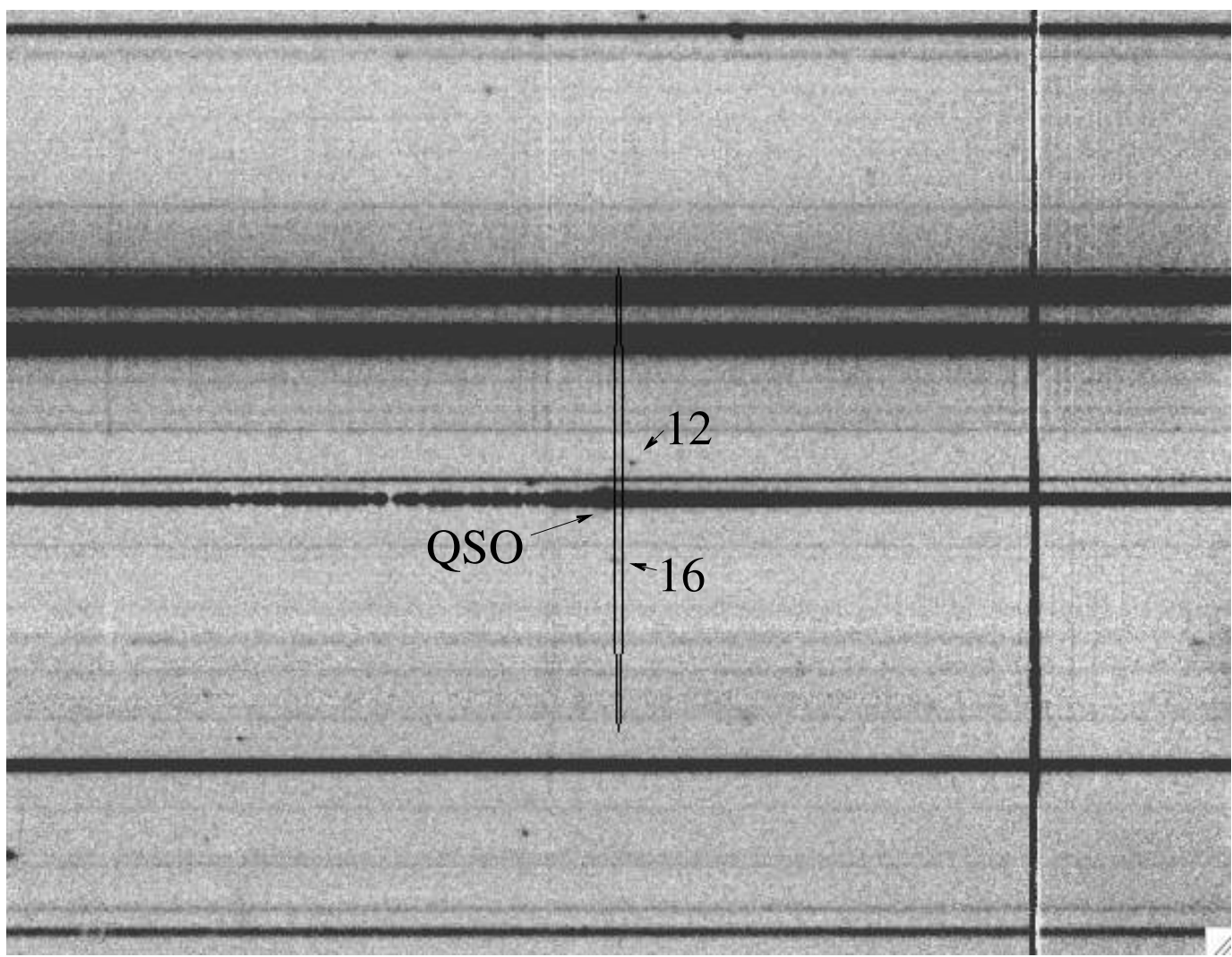

Fig. 16.-Elliptical "zone of influence" near the QSO where the ionizing radiation would be sufficient to cause fluorescence at the $10^{-18} \mathrm{erg} \mathrm{cm}^{-2} \mathrm{~s}^{-1} \mathrm{arcsec}^{-2} \mathrm{sur}-$ face brightness level.

check whether the observables agree at an order-of-magnitude level. As far as we can tell given the limited spatial resolution, our typical surface brightness profile requires that the sources are at least somewhat centrally concentrated, similar to model 4 of Dijkstra et al. (see the surface brightness profile in their Fig. 5). Our median observed "radius" (= half the extent along the slit) at the $10^{-19} \mathrm{erg} \mathrm{cm}^{-2} \mathrm{~s}^{-1} \operatorname{arcsec}^{-2}$ surface brightness contour is about $1.0^{\prime \prime}$, the median power-law slope $\alpha_{\text {med }}=-2.0$. Making

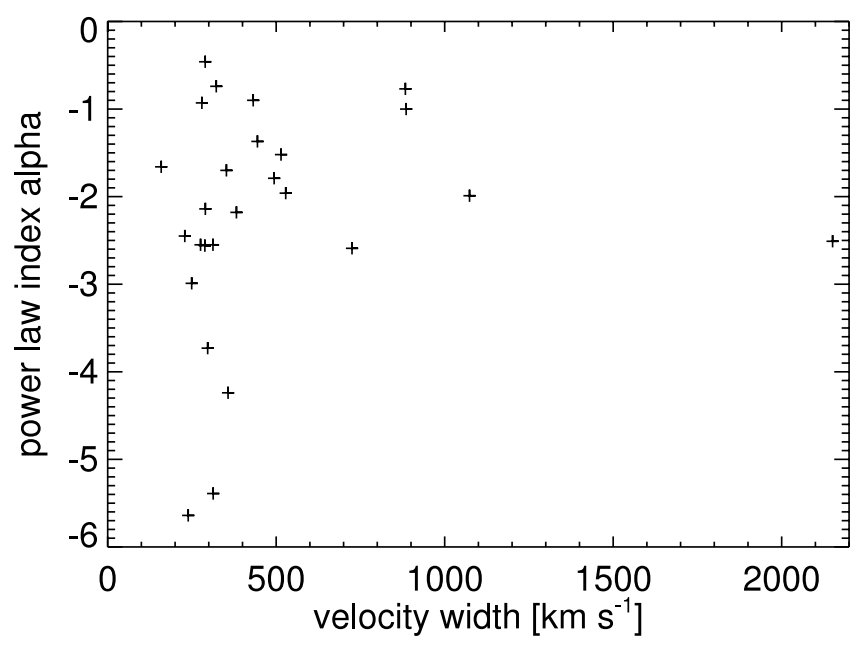

FIG. 17.-Power-law slope for the Gaussian+power law fit to the spatial surface brightness profile, vs. Gaussian velocity width of the emission line. There is no significant correlation between the two, but the objects with the largest velocity widths also seem to have small power-law indices, i.e., the slowest radial decline in surface brightness. appropriate corrections for the slit losses and distortions of the surface brightness profile, we find good agreement with the surface brightness profile shape of Dijkstra et al. if we scale down their total flux to $1.1 \times 10^{-17} \mathrm{erg} \mathrm{cm}^{-2} \mathrm{~s}^{-1}$. The mass dependence of the flux for their model 4 at $z=3.2$ is $4.16 \times 10^{-18}\left(M_{\text {tot }} /\right.$ $\left.10^{11} M_{\odot}\right)^{5 / 3} \mathrm{erg} \mathrm{cm}^{-2} \mathrm{~s}^{-1}$. Our corrected median flux, $1.1 \times$ $10^{-17} \mathrm{erg} \mathrm{cm}^{-2} \mathrm{~s}^{-1}$, would then correspond to a cooling halo with total mass $\sim 1.8 \times 10^{11} M_{\odot}$.

Thus, the median surface brightness profiles and total fluxes observed appear broadly consistent with the Ly $\alpha$ arising predominantly as cooling radiation. The typical halo mass required to produce the luminosity function of our emitters, is, however, uncomfortably large for cooling radiation to be the dominant source of Ly $\alpha$ for a majority of our objects. Dijkstra et al. (2006) estimate the expected cooling radiation assuming that the gas in DM halos cools on a free-fall timescale and that the cooling is predominantly by $\operatorname{Ly} \alpha$ emission. The Ly $\alpha$ emission is then a strong function of the virial velocity of the halo, $L_{\mathrm{Ly} \alpha} \sim 1.6 \times$ $10^{39}\left(v_{c} / 35 \mathrm{~km} \mathrm{~s}^{-1}\right)^{5} \mathrm{erg} \mathrm{s}^{-1}$. Note that the free-fall timescale is shorter than the time that corresponds to the redshift interval $2.667<z \leq 3.751$, and a newly collapsed DM halo would only emit for about $40 \%$ of the redshift range where we can observe it. Even if we assume that all DM halos present at the lower end of the redshift interval have collapsed and started cooling in our redshift interval, all halos with $v_{c}>35 \mathrm{~km} \mathrm{~s}^{-1}$ would be necessary to account for the observed space density of emitters. The Ly $\alpha$ luminosity for the typical object would generally be more than a factor of 10 lower than we observe even if we neglect the slit losses. This does not preclude that the flux of a few of our emitters in more massive halos is dominated by Ly $\alpha$ cooling radiation, but it is very unlikely that this is a large number. Ly $\alpha$ 


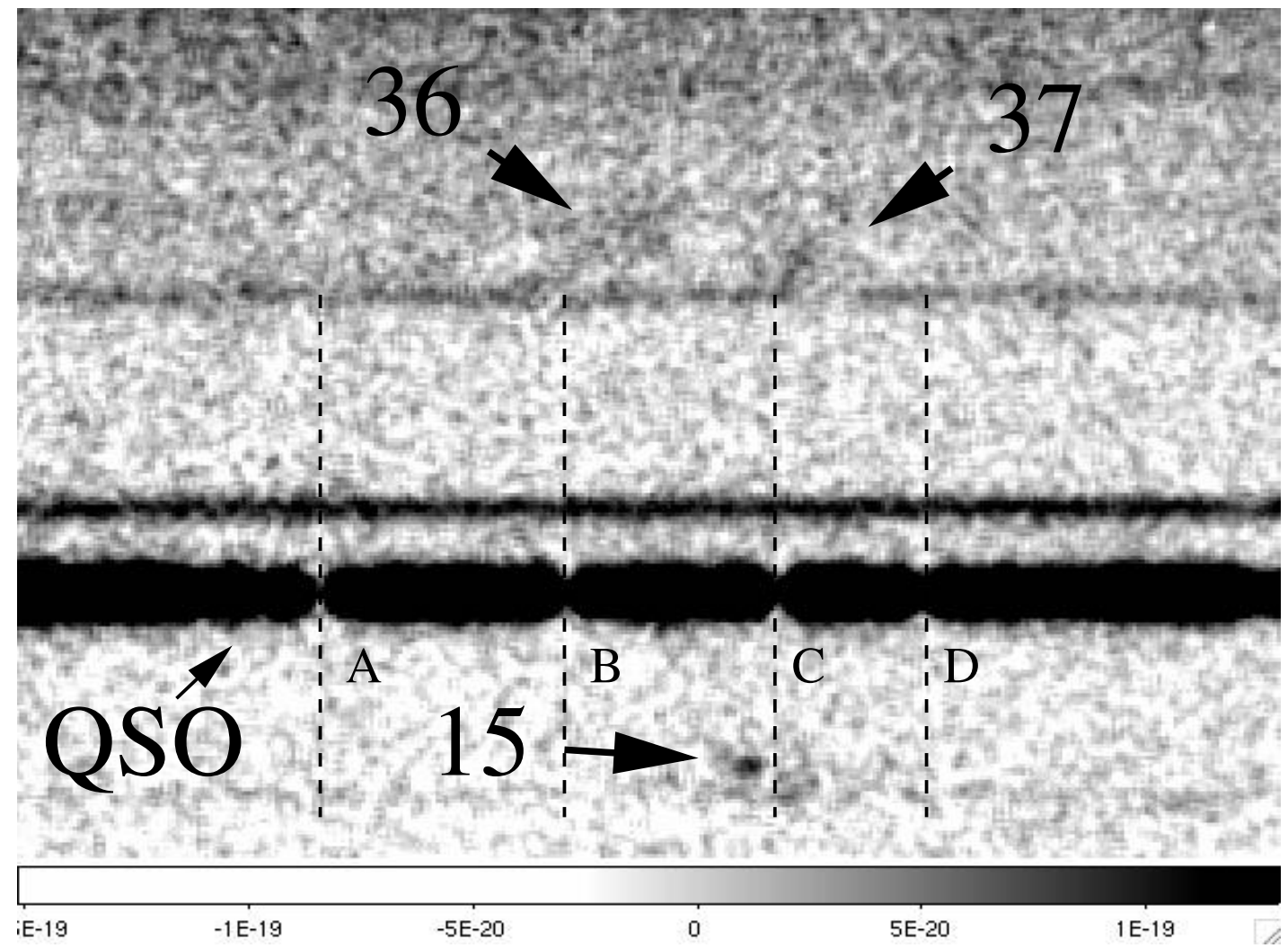

FIG. 18.-Immediate surroundings of smudges 36, 37, and 15. The labels A, B, C, and D refer to the strong absorption lines in the QSO spectrum, partly lining up with the emitters (see text). The featureless continuum object just above the QSO is a low-redshift object; the fainter continuum further up appears to show absorption by the emitting smudges 36 and 37.

radiation powered by star formation appears to be the energetically most favorable explanation for the majority of our emitters.

\subsubsection{Evidence for Radiative Transfer Mechanisms from Spectral Line Profiles}

Irrespective of the origin of $\operatorname{Ly} \alpha$ photons, radiative transfer of line photons from a central source within an optical thick halo should have observational signatures characteristic of the kinematics of the gas.

Several of our objects $(3,12,21,23,28,29$, and 39; see Figs. 3 and 4 ) exhibit strong, spatially concentrated emission peaks (even though their emission often extends farther out), with asymmetric line profiles showing a steep drop in the blue and an extended red shoulder; such profiles have been seen previously in low- and high-redshift star-forming galaxies and are generally considered to be consistent with radiative transfer in the expanding supershells of galactic winds (e.g., Lequeux et al. 1995; Mas-Hesse et al. 2003). At various stages of their evolution, the line profiles may resemble single emission line peaks, P Cygni profiles, or double-component profiles with a dominant red component (Tenorio-Tagle et al. 1999; Ahn et al. 2003; Ahn 2004).

Several of these asymmetric emitters $(3,23,28$, and 29$)$ show a weaker blue peak opposing the red one, which may be evidence for a wind shell or more generally radiative transfer through an expanding optically thick medium (Zheng \& Miralda-Escudé 2002; Dijkstra et al. 2006; Tasitsiomi 2006).

\subsubsection{Individual Candidates for Emitters Dominated by Cooling Radiation}

Most other emission profiles look amorphous and defy classification because of the low $\mathrm{S} / \mathrm{N}$ level, but there is a small group of emitters (objects 15, 36 and 37; Fig. 18) fortuitously projected near the QSO trace, which show a number of intriguing properties different from those of the other sources. The three continuum traces visible in that figure are the QSO (with four strong Ly $\alpha$ forest absorption lines of rest equivalent widths $2.0 \AA[\mathrm{A}], 1.3 \AA$ [B], $1.2 \AA[\mathrm{C}]$, and $0.9 \AA[\mathrm{D}])$; an unrelated, featureless, presumably low-redshift continuum object just above the QSO trace; and farther up a faint high-redshift object from which two faint emission smudges (36 and 37) seem to protrude. Even though their appearance seems unusual, the identification of all three smudges with $\mathrm{H}_{\mathrm{I}} \mathrm{Ly} \alpha$ is relatively secure, because of their close alignment in redshift with QSO absorption systems: the dip in the emission region of object 15 and the blue starting point of the emission region of object 37 both coincide to within less than $100 \mathrm{~km} \mathrm{~s}^{-1}$ with the strong absorption line $\mathrm{C}$ in the QSO spectrum between them. The projected transverse (here vertical) distances from the QSO are 150 (object 37) and 90 (object 15) physical kpc. Object 36, at a similar transverse distance from the QSO as 37, also coincides closely in redshift with another QSO absorption system (B).

Object 15, below the QSO trace, appears to consist of a strong blue emission component, separated by a dip in flux from a fuzzy redder emission bit, which also may be rotated slightly. The velocity shift between the blue emission line and the dip, and the FWHM of the blue peak, each amount to approximately $200 \mathrm{~km} \mathrm{~s}^{-1}$. It is difficult to be sure of what we are seeing here (perhaps two merging protogalactic clumps), but the signature of a strong blue peak, central dip, and weak red peak is not unlike that expected for cooling radiation from gas falling into a galactic halo. If this is what we are seeing, then, according to the simulations by Dijkstra et al. (their Fig. 8), such relatively small values for blueshift and FWHM of the blue peak may indicate a 
cooling halo with relatively small infall velocities and $\mathrm{H}$ i optical depths.

Object 37 (cf. Figs. 3 and 4) shows a barlike emission region projecting out at about a $60^{\circ}$ angle on one side from the blue edge of a strong absorption line in the nearby, faint background galaxy, as if a "door" had been opened anticlockwise in the continuum of that galaxy. The transverse extent of the emission is at least about $3.3^{\prime \prime}$, or 26 physical kpc. The spectral width of the tilted emission bar is about $320 \mathrm{~km} \mathrm{~s}^{-1}$ (i.e., it is possibly unresolved), and it projects out from the continuum object, starting about $490 \mathrm{~km} \mathrm{~s}^{-1}$ blueward of the centroid of the absorption line (rest equivalent width $3.9 \AA$ ) in the faint continuum object, shifting to the red with increasing distance from the continuum object by between 280 and $470 \mathrm{~km} \mathrm{~s}^{-1}$ (the uncertainty arises from the difficulty of estimating the spatial extent of the emission region).

Object 36 is another broad smudge of emission, loosely (the $\mathrm{S} / \mathrm{N}$ is poor) lining up in redshift with QSO absorption system $\mathrm{B}$ (a weak absorption feature appears in the continuum object, about $110 \mathrm{~km} \mathrm{~s}^{-1}$ blueward of the QSO absorber). Again, going outward from the continuum object, the emission can be traced spatially to a similar extent as object 37 , but in this case extends over a larger wavelength range, becoming redder by up to $1500 \mathrm{~km} \mathrm{~s}^{-1}$. The feature is clearly resolved in velocity, with a width of about $1000 \mathrm{~km} \mathrm{~s}^{-1}$.

All three objects show emission blueward of the absorption centroids of either the QSO absorption lines or the absorption in the continuum emitter. In addition, the color gradient from red to blue when approaching the absorption systems could be understood in terms of infalling halo gas, which accelerates and cools when approaching smaller radii, as described by Dijkstra et al. However, it is not clear that the absorption systems really represent the centroids of the halos and do not rather arise in the outskirts. A scenario with out- rather than inflows and a different topology cannot be excluded, at least not for objects 36 and 37 .

There are two more QSO absorbers in that group that span a total (from A to D) of $52.75 h^{-1} \mathrm{Mpc}$ (or 20 physical Mpc), if the redshift difference is due to the Hubble flow. The fact that the three most unusual emitters, together with a cluster of strong QSO absorption lines, occur in a spatially relatively narrowly but apparently highly elongated region (even the line-of-sight distance between $\mathrm{B}$ and $\mathrm{C}$ is 7 physical Mpc, or $18.43 h^{-1}$ comoving $\mathrm{Mpc}$ ) suggests that we may be looking along a large-scale filament or sheet, with the QSO absorbers representing the outskirts of the three galaxies whose emission regions we see.

\section{CORRESPONDENCE BETWEEN THE LINE EMITTERS AND OPTICALLY THICK Ly $\alpha$ FOREST ABSORPTION SYSTEMS}

The existence of two independently identified classes of optical thick objects in the universe, Ly $\alpha$ emitters and Lyman limit absorption systems, enables us to establish a correspondence between them and constrain their properties.

If we assume that the emitting and absorbing regions are identical in size (in reality, the absorption cross section may be an upper limit to the emission cross section, for optically thick gas), we can equate the rate of incidence per unit redshift, $d N_{\mathrm{LL}} / d z$, of Lyman limit absorbers above a certain $\mathrm{H}$ I column density, and the product of the spatial comoving density of Ly $\alpha$ emitters, their emission cross section, and the redshift path,

$$
\frac{d N_{\mathrm{LL}}}{d z}=\sum_{i} \frac{\sigma_{i}}{V_{i}} \frac{d l}{d z},
$$

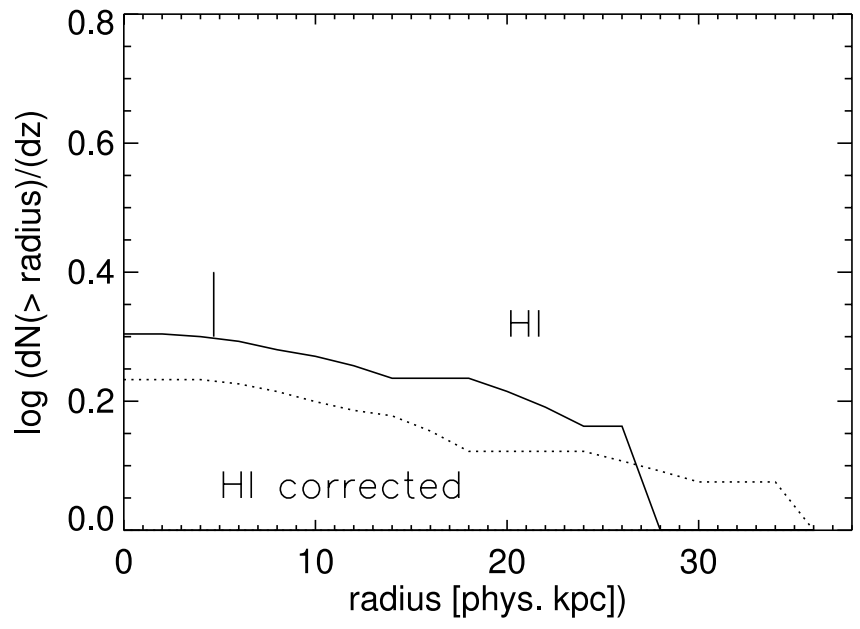

FIG. 19.-Contribution of objects of different sizes to the rate of incidence per unit redshift, $d N / d z$, for $\mathrm{H}$ I with (dotted line) and without (solid line) correction for the extended sizes and our underestimating the radius. The short vertical line riding on top of the uncorrected curve indicates the spatial resolution limit along the slit.

where

$$
\frac{d l\left(z_{i}\right)}{d z}=\frac{c}{H_{0} E\left(z_{i}\right)} .
$$

The comoving density of objects is again $d N / d V_{c}=\mathcal{N}_{\mathrm{obj}} /$ $\left(885 h_{70}^{-3} \mathrm{Mpc}\right)$. From Figure 19, our total observed rate of incidence of Ly $\alpha$ emitters would be $d N / d z=0.30$, if all 27 objects were Ly $\alpha$ emitters.

They same cautions as mentioned above about slit losses apply to the estimate of the radius from the size along the slit. If the emitters had a spherical, sharp-edged outline in the plane of the sky, then by adopting the total extent along the slit for the diameter of the object we would underestimate the latter by a factor of $\pi / 4$. The finite sizes of the objects also affect the detectability on the slit, as an extended object may be detected even if its center falls outside the slit. This increases the effective comoving volume and decreases the space density for the objects. We use a simple model to compute the comoving volume, where an object of a given radius can be detected if part of it fills the slit. Our correction can only be indicative of the true corrections. The little we know about the emitters at present does not warrant a more detailed approach.

The resulting $d N / d z$, corrected for these effects, is shown in Figure 19 as a dotted line. The correction emphasizes the relative contributions to $d N / d z$ from objects smaller than the slit width, and reduces the relative contribution from larger objects. The total correction (larger cross section and smaller comoving volume) reduces the overall $d N / d z$ by about $22 \%$, to $d N / d z=0.23$. About half of the contribution to $d N / d z$ arises from the four most extended objects.

Interestingly, the observed $d N / d z$ for our emitters and the one for DLAS with neutral hydrogen column densities $N_{\mathrm{H}_{\mathrm{I}}}>2 \times$ $10^{20} \mathrm{~cm}^{-2}\left(d N / d z_{\text {DLA }}=0.26\right.$; Peroux et al. 2005; Storrie-Lombardi $\&$ Wolfe 2000) are comparable.

In other words, the combination of large sizes and the high space density of the emitters together imply that the total rate of incidence is sufficient to explain the majority of damped Ly $\alpha$ systems. It thus appears that we may have finally detected the star formation associated with most of the rate of incidence of damped Ly $\alpha$ absorption systems in the redshift range $2.667<z<3.751$. 
The low star formation rates of $0.07-1.5 M_{\odot} \mathrm{yr}^{-1}$ would explain the low success rate of direct searches for the host galaxies of DLAS. If the interpretation of the emission as being due to the hosts of DLAS is correct, then we have for the first time established the typical size and space density of DLA host galaxies. Within the CDM model of structure formation, we can then also infer their masses $\left(3 \times 10^{10} M_{\odot}\right.$ total, $5 \times 10^{9} M_{\odot}$ in baryons $)$ and virial velocity scale $\left(\sim 50 \mathrm{~km} \mathrm{~s}^{-1}\right)$. The typical values for size, mass, and virial velocity agree well with the predictions of the model of Haehnelt et al. $(1998,2000)$, who interpreted the observed kinematic properties of the neutral gas in DLAS as probed by low-ionization species within the context of CDM models. The inferred star formation rates and the inferred masses are consistent with the low observed metallicities of DLAS, which are generally overpredicted in models assuming larger star formation rates. Note that if all the observed emission is due to Ly $\alpha$, the total star formation rate density corresponds already to $36\left(f_{\text {slit }} / 1.0\right) \%$ of the total non-dust-corrected star formation rate inferred from dropout studies at these redshifts (e.g., Bouwens et al. 2007; $f_{\text {slit }}$ is the factor by which the observed flux needs to be multiplied to correct for slit losses). Unfortunately, we have no direct observational handle to decide whether the star formation itself is extended. If all the emission is Lyo, our total star formation rate density is, however, higher by at least an order of magnitude than the upper limits obtained by Wolfe \& Chen (2006), who searched for $e x$ tended continuum emission from DLAS in the HUDF. At the same time, our star formation density is close to (about $60 \%$ of) the value needed to explain the heating of DLAS (Wolfe et al. 2003). As suggested by Wolfe et al., these discrepancies can be reconciled if the star formation in question is confined to a compact region at the center of DLA hosts, rather than arising in large stellar disks. The Ly $\alpha$ emission in our objects often appears extended, but this does not necessarily mean that the sources of the ionizing photons responsible for producing the Ly $\alpha$ photons are similarly large. The extended nature of the Ly $\alpha$ emission could be due to resonant line scattering, with Ly $\alpha$ photons random walking their way out to radii that have never seen a star. Alternatively, some of the large sizes could be due to unresolved merging protogalactic clumps, a situation that is common in a CDM scenario and may explain the observed kinematics of DLAS (Haehnelt et al. 1998). Finally, the identification of the emitters with DLAS, which are known to be essentially dust-free (e.g., Murphy \& Liske 2004), and the realization that Ly $\alpha$ from emitters brighter by an order of magnitude have Ly $\alpha$ emission reduced by a factor of 3, presumably by dust (e.g., Gronwall et al. 2007), would also explain at least in part the steep rise of the number of emitters over a decade in surface brightness as a drop in dust content.

\section{THE HOGAN-WEYMANN EFFECT}

So far we have not addressed the effect of Ly $\alpha$ fluorescence, induced by the general UV background (Hogan \& Weymann 1987). The original expectation was that the number of objects lit up by the UV background corresponding to optically thick LLS would yield about the same number of objects (30, with a radius of $2.5^{\prime \prime}$ at column density $10^{19} \mathrm{~cm}^{-2}$ ) as we have found, albeit at considerably lower surface brightness. A possible explanation is that many LLS may have ongoing local, low-level star formation. This would be consistent with LLS having somewhat higher metallicities (Steidel 1990) than the general Ly $\alpha$ forest (Simcoe et al. 2004). Given our surface brightness profiles, it is possible that the underlying fluorescence is simply swamped by star formation Ly $\alpha$.

There are several conceivable approaches to searching for lowlight-level emitters in the field. Originally we had considered the possibility of a blind search (e.g., Bunker et al. 1998), which, how-

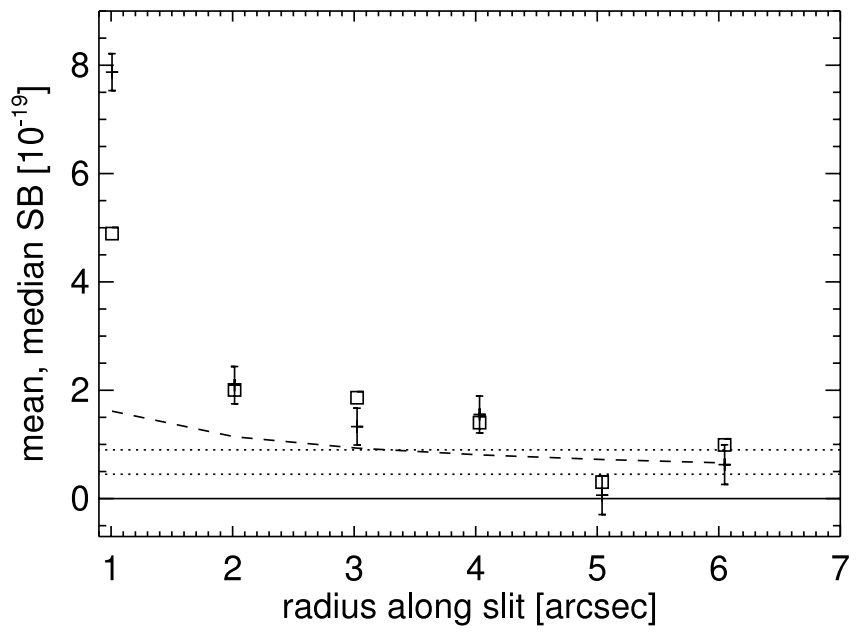

FIG. 20.-Mean ( points with error bars) and median (open squares) surface brightness measurements in units of $10^{-19} \mathrm{erg} \mathrm{cm}^{-2} \mathrm{~s}^{-1} \operatorname{arcsec}^{-2}$ for the combined surface brightness profiles, as a function of angular distance in arcseconds from the center of emission along the slit. The dotted lines give the range of the expected surface brightness based on the Bolton et al. (2005) photoionization rate of the $z \sim 3$ IGM. The upper dotted line is for a QSO type UV spectrum, and the bottom line is for a spectrum where $50 \%$ of the flux is contributed by galaxies. The dashed line is the $4 \sigma$ surface brightness detection threshold for individual objects.

ever would have reached only a sensitivity a factor of 2 above the recently revised (lower!) estimates for the anticipated signal, based on the opacity of the Ly $\alpha$ forest (Bolton et al. 2005). One possibility to increase the sensitivity, suggested by the number of objects already detected, is to search in their immediate neighborhood for an extended signal of diffuse emission surrounding the brighter star-forming regions that is in agreement with the expected surface brightness (Gould \& Weinberg 1996),

$9 \times 10^{-20}\left(\frac{\eta}{0.5}\right)\left(\frac{J}{4.3 \times 10^{-22}}\right) \operatorname{erg~cm}{ }^{-2} \mathrm{~s}^{-1} \operatorname{arcsec}^{-2}$,

at $\langle z\rangle=3.2$ for a QSO-dominated, Haardt \& Madau (1996) UV spectrum with slope $1.73 ; \eta$ is the fraction of the energy of the impinging UV photons converted into $\operatorname{Ly} \alpha$, and $J$ is the UV background. The value for $J$ in equation (24) is scaled to the recent measurement of the photoionization rate at $z \sim 3$ in the Ly $\alpha$ forest (Bolton et al. 2005). An ionizing spectrum in which galaxies and QSOs each produce half of the ionizing flux would result in a surface brightness lower by a factor of 2 than the fiducial value in equation (24).

Our observation is deep enough to probe the surface brightness for general fluorescence if we combine the signal from all sources to improve the signal-to-noise ratio. Boxes of spatial width $2^{\prime \prime}$ and spectral length $1500 \mathrm{~km} \mathrm{~s}^{-1}$ (to include the likely extent of the double-humped emission profiles predicted; e.g., Cantalupo et al. 2005) have been placed on either side of our emitters, at varying distances along the slit direction. The boxes were sky-subtracted once more locally, using windows below and above (along the slit) the box used for the signal, but farther out from the emitter than the signal boxes. Because of the strong presence of weak continuum objects in the two-dimensional spectrum, only a subset of the emitters (usually 12-14) were in a sufficiently clean area of the field to be useful for this analysis. The mean and median surface brightnesses and their statistical errors were extracted. The results are presented in Figure 20. The open squares show the medians, and the crosses with error bars give the total weighted mean surface brightness of the boxes used, 
in units of $10^{-19} \mathrm{erg} \mathrm{cm}^{-2} \mathrm{~s}^{-1} \operatorname{arcsec}^{-2}$, as a function of angular distance in arcseconds along the slit. We caution that the error bars shown are merely statistical noise errors and do not include the fluctuations of the sky level reflected in the difficulty of finding a "clean" patch of sky to place the background-subtraction windows on. The combined profile is not very meaningful in the innermost $2^{\prime \prime}$ because of the wide variation in amplitudes, but if there were a universal fluorescent glow we would expect the outskirts of the objects to take the appearance of annuli of uniform surface brightness. In practice, such a plateau should be washed out by the distribution in sizes of the emitters.

There is a hint of a flattening between $3^{\prime \prime}$ and $4^{\prime \prime}$, but the signal continues to dive beyond $5^{\prime \prime}$. However, at a level of $\sim 2 \times$ $10^{-19} \mathrm{erg} \mathrm{cm}^{-2} \mathrm{~s}^{-1} \operatorname{arcsec}^{-2}$, the surface brightness is rather higher, by a factor of $2-4$, than expected for fluorescent emission with the favored range of the UV background intensity. Close inspection of the frame shows that this signal appears to be due to some genuinely very extended individual objects, i.e., these are not artifacts of the seeing. This is consistent with the large extent of the $1.5 \times 10^{-19} \mathrm{erg} \mathrm{cm}^{-2} \mathrm{~s}^{-1}$ arcsec $^{-2}$ surface brightness contour, corresponding to a flux density of $1.5 \times 10^{-20} \mathrm{erg} \mathrm{cm}^{-2} \mathrm{~s}^{-1} \AA$ seen in Figure 3.

It is intriguing that the corresponding physical radius is $30 \mathrm{kpc}$, about 4 times larger than we had estimated based on the individually modeled surface brightness profiles above. If this were identical to the radius out to which all objects have optically thick $\mathrm{H}$ I, our sample of 27 emitters would project a $d N / d z \sim 1.4$, and would correspond to all LLS with column densities larger than about $3 \times 10^{18} \mathrm{~cm}^{-2}$, where the fraction of Ly $\alpha$ photons per ionizing photon is just flattening off to attain the maximum conversion rate (Gould \& Weinberg 1996). We will defer a more detailed analysis to future study, and conclude that the large lateral median extent of our emitters is fully consistent with them being surrounded by optically thick, Lyman limit zones that radiate to within a factor of 2 at the level predicted by Gould \& Weinberg (1996), updated by latest estimates for the photoionization rate.

\section{CONCLUSIONS}

Our long-slit search for Ly $\alpha$ fluorescence from the intergalactic medium, taking advantage of moderate seeing periods with FORS2 at the VLT, has yielded a sample of 27 faint emitters with line fluxes of a few $\times 10^{-18} \mathrm{erg} \mathrm{s}^{-1} \mathrm{~cm}^{-2}$ over a redshift range $2.66<z<3.75$.

At least a third of the sample show emission-line profiles or an association with absorption systems in the nearby QSO, strongly suggesting identification with $\operatorname{Ly} \alpha$. Spectroscopic features and the absence of detected continua down to $3 \sigma$ flux limits of $\sim 1.5 \times$ $10^{-19} \mathrm{erg} \mathrm{s}^{-1} \mathrm{~cm}^{-2}$ make a direct identification of the other emitters (as $\mathrm{H}$ I Ly $\alpha$, [O II] doublet, or $[\mathrm{O}$ III] $/ \mathrm{H}$ I Balmer emission lines) difficult, but comparison with known galaxy populations and other statistical arguments indicate that the majority of emitters are likely to be $\operatorname{Ly} \alpha$ at mean redshift 3.2.

If this identification is correct, the emitters present a steeply rising luminosity function with a total number density more than 20 times larger than the comoving density of Lyman break galaxies $\left(M_{R}<25.5\right)$ at comparable redshifts. About half of the profiles are extended, possibly owing to radiative transfer of Ly $\alpha$ photons from a central source, and there are candidates for both outflows and infall features. We have investigated several mechanisms for the Ly $\alpha$ production and find star formation to be the energetically most viable process, with a few objects being candidates for cooling radiation.

The inferred low star formation rates, large line-emission cross sections, high number density, and a fitting total cross section per unit redshift on the sky seem to provide an excellent match to the low luminosities, low metallicities, low dust content, and rate of incidence of damped Ly $\alpha$ systems, the main reservoir of neutral gas at high redshift. This suggests that our objects are the longsought counterparts of DLAS in emission. The properties of the objects paint the DLAS host galaxies as a population of lowmass protogalactic clumps, as suggested by some of us (Haehnelt et al. 1998, 2000; Rauch et al. 1997b), disfavoring a model dominated by large disk galaxies (Prochaska \& Wolfe 1997). Recent, apparently contradictory limits on spatially extended star formation from DLAS (Wolfe \& Chen 2006) and on the heating of DLAS (Wolfe et al. 2003) are consistent with the amount of star formation we measure if it is confined to a small unresolved region, irrespective of the fact that both the absorption and the Ly $\alpha$ emission cross sections appear much larger, the latter because of the random walk of photons to the edge of optically thick gas. The physical origin and the nature of the extended optically thick gas is uncertain, but it is intriguing that quite a few objects show Ly $\alpha$ emission-line profiles consistent with galactic outflows. Thus, the absorption cross section of DLAS could be enhanced by winds, a possibility raised by Nulsen et al. (1998) and discussed in the context of Lyman break galaxies by Schaye (2001).

Finally, adding up the surface brightness profiles of all objects in the outer $2^{\prime \prime}-6^{\prime \prime}$, we detect radiation at the $>2 \times 10^{-19} \mathrm{erg} \mathrm{cm}^{-2}$ $\mathrm{s}^{-1} \operatorname{arcsec}^{-2}$ level out to $4^{\prime \prime}$. The light level is higher by a factor of 2-4 than the Ly $\alpha$ fluorescence signal expected for a UV background intensity consistent with current estimates for the photoionization rate of the intergalactic medium. The large sizes can be explained if radiative transfer of Ly $\alpha$ lights up the outskirts of our objects out to a radius where the conversion from UV to Ly $\alpha$ starts to become inefficient. A radius of $4^{\prime \prime}$ combined with the observed comoving density of our sample can explain the rate of incidence of DLAS and LLS with column densities as low as $3 \times 10^{18} \mathrm{~cm}^{-2}$, consistent with the possibility that many LLS arise in the outskirts of DLAS, which in turn are to be identified with the faint emitters. With our interpretation, the gas in DLAS is the reservoir from which typical $L_{*}$ galaxies must have formed in a CDM-based universe.

Some of our conclusions here are speculative. Further study is highly desirable, but in any case it should be obvious that long spectroscopic exposures are a promising way of discovering lowmass galaxies. Performing single-field, blank-sky searches for Ly $\alpha$ emitters for longish amounts of observing time (but not longer than regularly used by radio astronomers and now even in space-based UV, optical, and X-ray astronomy) can bring a whole new range of astrophysical phenomena within the range of existing groundbased, optical telescopes, and obviously, could provide one of the most exciting science projects for a future generation of ultralarge telescopes.

We would like to thank the following people for helpful advice and discussions during the course of this project: James Bolton, Scott Burles, Bob Carswell, Hsiao-Wen Chen, Sandro D’Odorico, Johan Fynbo, Rob Kennicutt, Juna Kollmeier, Rob Simcoe, and Neil Trentham. We thank the Director General of ESO, Catherine Cesarsky, for keeping this project afloat, and we are grateful to the observatory staff at ESO and Gemini for performing the observations, and to the time assignment committees of both observatories. M. R. thanks the IoA for hospitality in spring and summer 2007. G. D. B. and M. R. were supported by the National Science Foundation through grant AST 05-06845. This work was partially supported by the EC RTN network "The Physics of the Intergalactic Medium." 


\section{APPENDIX A}

\section{SLIT LOSSES AND SELECTION EFFECTS}

The determination of total fluxes and surface brightnesses from long-slit spectra is generally not possible for individual objects, the problem being the unknown spatial shape and overall extent, and the random position of the slit center relative to the position of the underlying object in the plane of the sky. The spatial variation of the surface brightness along the slit is bound to generally be different from the intrinsic surface brightness dependence on radius. However, performing simulations with a given radial surface brightness distribution, size, and radial symmetry, one can obtain an impression of the average slit losses and of the relation between the actual
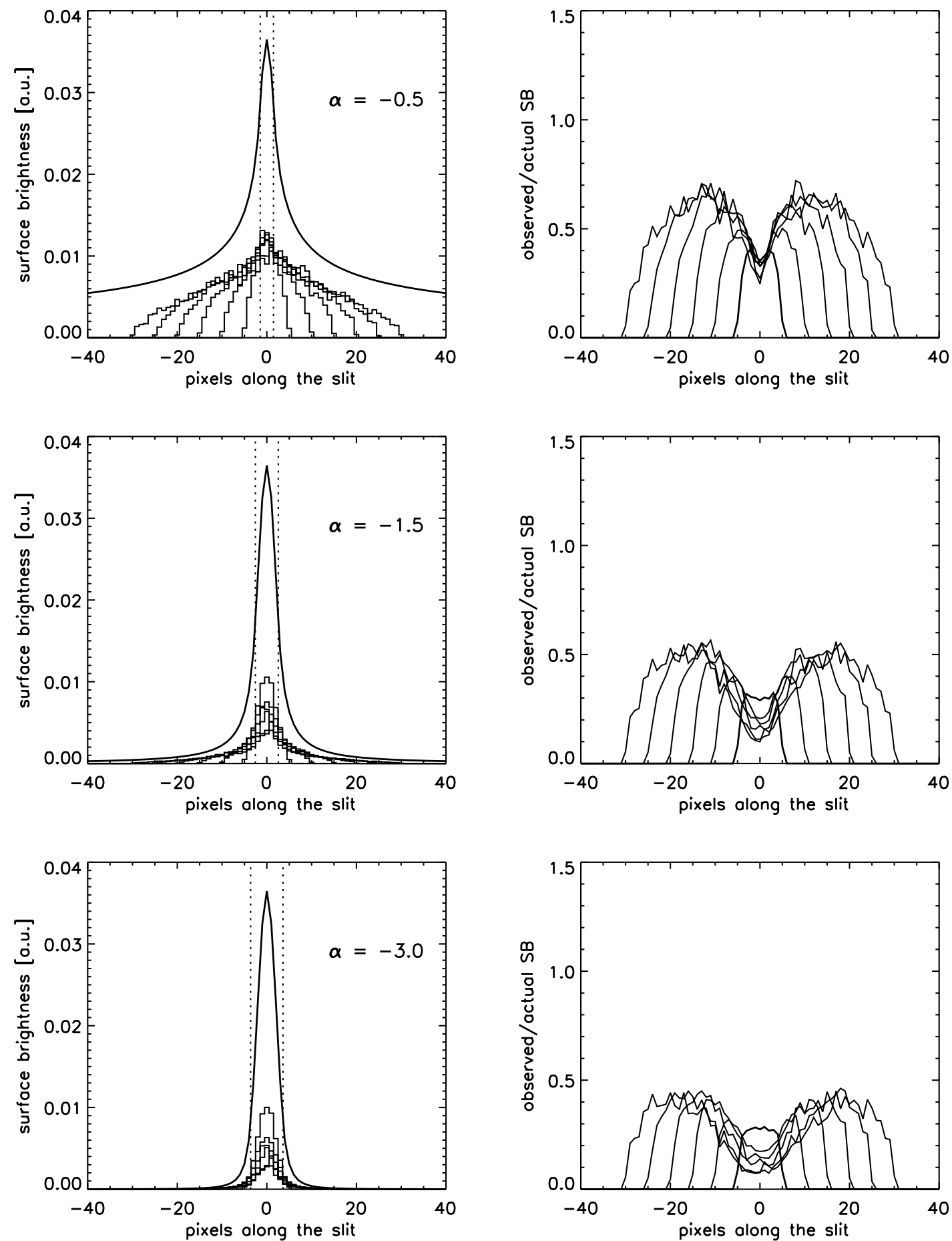

FIG. 21.- Simulation of slit losses. Left column: Radial surface brightness profile of the input model (smooth curve) and "observed" average profiles (binned curves)

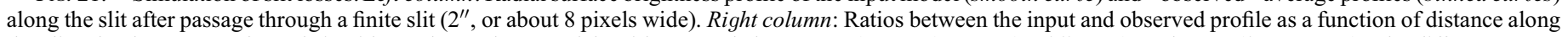

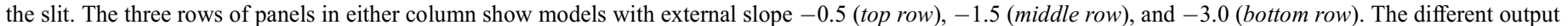

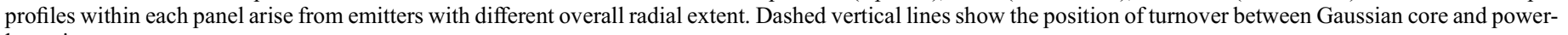
law wings. 


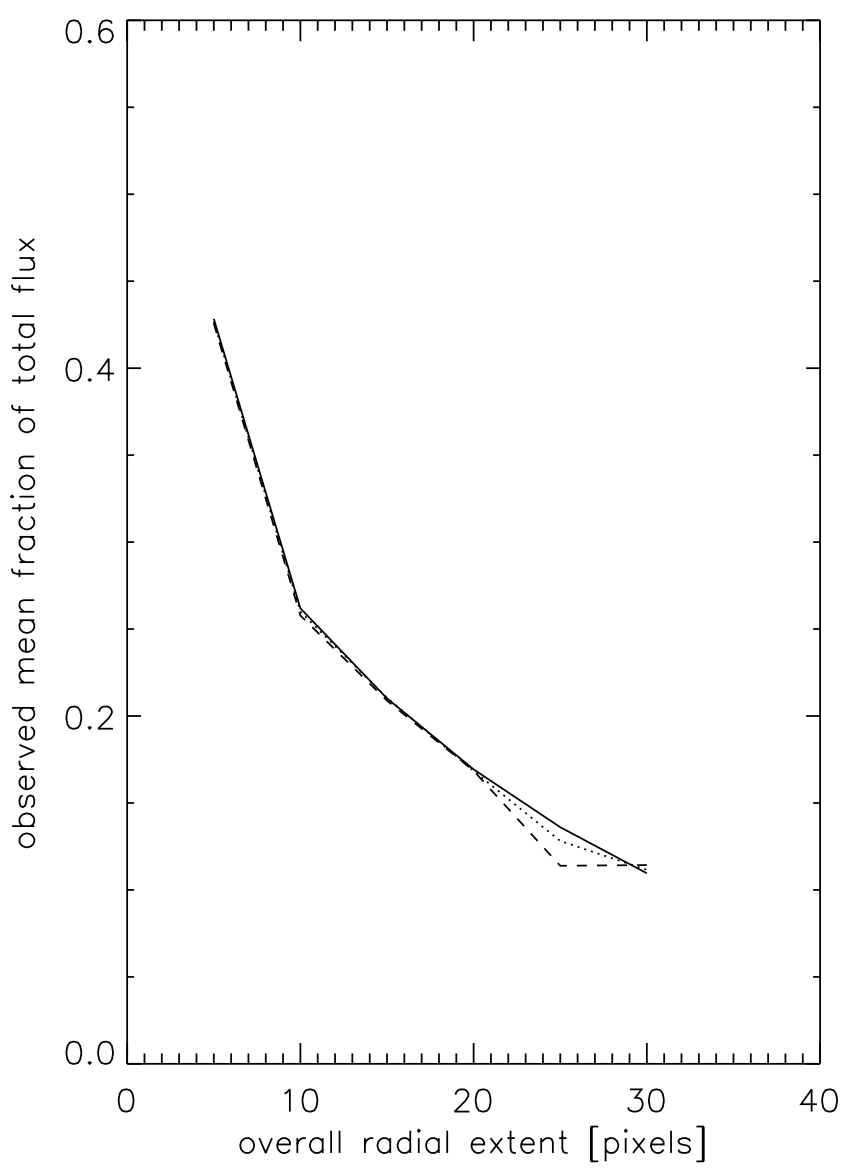

FIG. 22.-Average fraction of total flux passing through the $2^{\prime \prime}(8$ pixel) wide slit, as a function of total radial extent of the emitter.

radial surface brightness dependence and the typical distribution of surface brightness as a function of position along the slit. The results of Monte Carlo simulations of a long slit $2^{\prime \prime}$ wide, randomly positioned over a set of disks with a given size and surface brightness dependence, are shown in Figures 21 and 22. The model distribution assumes the surface brightness to have a Gaussian core with a width to match the measured seeing, replaced by a power law at larger radii:

$$
S(r) \propto \begin{cases}\exp \left[-r^{2} /\left(2 \sigma^{2}\right)\right] & \text { for } r<r_{\mathrm{turn}}, \\ r^{\alpha} & \text { for } r>r_{\mathrm{turn}} .\end{cases}
$$

For simplicity, the transition between the Gaussian and the power law at the turnover radius, $r_{\text {turn }}$, was chosen such that the amplitude and slope of the two regimes were continuous at $r_{\text {turn }}$, corresponding to $r_{\text {turn }}=(-\alpha \sigma)^{1 / 2}$.

Figure 21 shows a number of radial model surface brightness distributions (smooth curves) and the predicted average observed distributions (binned curves) along a long slit $2^{\prime \prime}$ wide, derived from a Monte Carlo simulations with 100 realizations (= random slit positions) per model. For the input distribution, the abscissa in all panels is the radius from the center of the emitter; for the output predicted surface brightness, it is the spatial coordinate along the slit (both in units of $0.252^{\prime \prime}$ wide pixels). Note that for nontrivial functional forms these distributions should not agree unless the slit is infinitely narrow and runs precisely radially with respect to the underlying emitter.

The left panels show the form of these distributions for three different external power law slopes $\alpha=-0.5$ (top), -1.5 (middle), and -3.0 (bottom), representing profiles increasingly dominated by a central peak. The right panels show the ratios between the same input radial surface brightness distributions and the predicted "along-the-slit" distributions. The multiple curves in each panel represent emitters with different overall radial extent, going from $R=5$ to 30 pixels in steps of 5 . With a pixel scale of $0.252^{\prime \prime}$, the largest emitter model considered would then be $30 \times 0.252^{\prime \prime} \approx 7.5^{\prime \prime}$ in radius. The dotted vertical lines in the left panels show the location of the transition radius between the central Gaussian and external power law. The right panels show the ratios between input radial and output slit distribution. From the right panels it is clear that the observed surface brightness along the slit is significantly distorted from the actual radial distribution. In particular, the central peak and the outer edges are suppressed in the observed profile, in both cases because they do not subtend a large area and are difficult to hit with a randomly positioned slit. Besides, the overall surface brightness is depressed typically by a factor of 2 or more.

Figure 22 shows the dependence of the average total flux received through the slit as a function of the radius of the underlying emitter (again in pixels). The spectrum contains about $43 \%$ of the flux for a source cut off radially at 5 pixels $\left(\sim 1.25^{\prime \prime}\right)$, but only $20 \%$ for a source with an extent of 16 pixels $\left(\sim 4^{\prime \prime}\right)$. Thus, within the $R=4^{\prime \prime}$ where there is detectable flux in some of the sources discovered here, we would underestimate the total flux by up to a factor of 5 . 
When applied to a luminosity function, this upward correction in flux by a factor of $2-5$ will not affect the numbers of emitters in the faint bins (say, below $F=10^{-18}$ ), because the cumulative distribution is flat (incomplete) here anyway, but it will increase each individual luminosity in the brighter bins, leading to a larger abundance of relatively bright objects, and will just shift the abundance of emitters to a flux range brighter by a factor of $2-5$.

Adams, T. F. 1972, ApJ, 174, 439

Adelberger, K. L., Steidel, C. C., Kollmeier, J, A., \& Reddy, N. A. 2006, ApJ, 637,74

Ahn, S.-H. 2004, ApJ, 601, L25

Ahn, S.-H., Lee, H.-W., \& Lee, H. M. 2003, MNRAS, 340, 863

Beckwith, S. V. W., et al. 2006, AJ, 132, 1729

Binette, L., Wang, J. C. L., Zuo, L., \& Magris, C. G. 1993, AJ, 105, 797

Bolton, J. S., Haehnelt, M. G., Viel, M., \& Springel, V. 2005, MNRAS, 357, 1178

Bouwens, R. J., Illingworth, G. D., Franx, M., \& Ford, H. 2007, ApJ, 670, 928

Bower, R. G., et al. 2004, MNRAS, 351, 63

Brocklehurst, M. 1971, MNRAS, 153, 471

Bunker, A., Smith, J., Spinrad, H., Stern, D., \& Warren, S. 2003, Ap\&SS, 284, 357

Bunker, A. J., Marleau, F. R., \& Graham, J. R. 1998, AJ, 116, 2086 1999a, in Lecture Notes in Physics, Galaxies in the Young Universe II Workshop, ed. H. Hippelein \& K. Meisenheimer (Berlin: Springer) (astroph/9912565)

Bunker, A. J., Stanway, E. R., Ellis, R. S., \& McMahon, R. G. 2004, MNRAS, 355,374

Bunker, A. J., Warren, S. J., Clements, D. L., Williger, G. M., \& Hewett, P. C. 1999b, MNRAS, 309, 875

Cantalupo, S., Lilly, S. J., \& Porciani, C. 2005, ApJ, 628, 61 . 2007, ApJ, 657, 135

Chen, H.-W., Kennicutt, R. C., \& Rauch, M. 2005, ApJ, 620, 703

Christensen, L., Wisotzki, L., Roth, M. M., Sanchez, S. F., Kelz, A., \& Jahnke, K. 2007, A\&A, 468, 587

Chun, M. R., Gharanfoli, S., Kulkarni, V. P., \& Takamiya, M. 2006, AJ, 131, 686

Cowie, L. L., \& Hu, E. M. 1998, AJ, 115, 1319

Dey, A., et al. 2005, ApJ, 629, 654

Dijkstra, M., Haiman, Z., \& Spaans, M. 2006, ApJ, 649, 14

Erb, D. K., Steidel, C. C., Shapley, A. E., Pettini, M., Reddy, N. A., \& Adelberger, K. L. 2006, ApJ, 647, 128

Fardal, M. A., Katz, N., Gardner, J. P., Hernquist, L., Weinberg, D. H., \& Dav, R. 2001, ApJ, 562, 605

Francis, P. J., \& Bland-Hawthorn, J. 2004, MNRAS, 353, 301

Francis, P. J., \& McDonnell, S. 2006, MNRAS, 370, 1372

Francis, P. J., et al. 2001, ApJ, 554, 1001

Franx, M., Illingworth, G. D., Kelson, D. D., van Dokkum, P. G., \& Tran, K.-V. 1997, ApJ, 486, L75

Fujita, S. S., et al. 2003, AJ, 125, 13

Furlanetto, S. R., Schaye, J., Springel, V., \& Hernquist, L. 2005, ApJ, 622, 7

Fynbo, J. P. U., Ledoux, C., Möller, P., Thomsen, B., \& Burud, I. 2003, A\&A, 407,147

Fynbo, J. U., Møller, P., \& Warren, S. J. 1999, MNRAS, 305, 849

Gerhard, O., Arnaboldi, M., Freeman, K. C., Okamura, S., Kashikawa, N., \& Yasuda, N. 2005, ApJ, 621, L93

2007, A\&A, 468, 815

Gould, A., \& Weinberg, D. H. 1996, ApJ, 468, 462

Gronwall, C., et al. 2007, ApJ, 667, 79

Haardt, F., \& Madau, P. 1996, ApJ, 461, 20

Haehnelt, M. G., Steinmetz, M., \& Rauch, M. 1998, ApJ, 495, 647

2000, ApJ, 534, 594

Haiman, Z., \& Rees, M. J. 2001, ApJ, 556, 87

Haiman, Z., Spaans, M., \& Quataert, E. 2000, ApJ, 537, L5

Hall, P. B., Osmer, P. S. Green, R. F., Porter, A. C., \& Warren, S. J. 1996, ApJ, 462,614

Hogan, C. J., \& Weymann, R. J. 1987, MNRAS, 225, 1

Hogg, D. W., Cohen, J. G., Blandford, R., \& Pahre, M. A. 1998, ApJ, 504, 622

Hu, E. M., Cowie, L. L., \& McMahon, R. G. 1998, ApJ, 502, L99

Johansson, P. H., \& Efstathiou, G. 2006, MNRAS, 371, 1519

Kauffmann, G. 1996, MNRAS, 281, 475

Keel, W. C., Cohen, S. H., Windhorst, R. A., \& Waddington, I. 1999, AJ, 118, 2547

Kelson, D. D. 2003, PASP, 115, 688

Kennicutt, R. C. 1998, ApJ, 498, 541

Kudritzki, R.-P., et al. 2000, ApJ, 536, 19
, V. P., Hill, J. M., Schneider, G. Weymann, R. J., Storrie-Lombardi, L. J., Rieke, M. J., Thompson, R. I., \& Jannuzi, B. T. 2000, ApJ, 536, 36 . 2001, ApJ, 551, 37

LeBrun V., Bergeron, J., Boissé, P., \& Deharveng, J. M. 1997, A\&A, 321, 733

Le Delliou, M., Lacey, C., Baugh, C. M., Guiderdoni, B., Bacon, R., Courtois, H., Sousbie, T., \& Morris, S. L. 2005, MNRAS, 357, L11

Le Delliou, M., Lacey, C. G., Baugh, C. M., \& Morris, S. L. 2006, MNRAS, 365,712

Ledoux, C., Petitjean, P., Bergeron, J., Wampler, E. J., \& Srianand, R. 1998, A\&A, 337, 51

Lequeux, J., Kunth, J. M., Mas-Hesse, J. M., \& Sargent, W. L. W. 1995, A\&A, 301,18

Lowenthal, J., D. Hogan, C. J., Leach, R. W., Schmidt, G. D., \& Foltz, C. B. 1990, ApJ, 357, 3

Madau, P., Pozzetti, L., \& Dickinson, M. 1998, ApJ, 498, 106

Mas-Hesse, J. M., Kunth, D., Tenorio-Tagle, G., Leitherer, C., Terlevich, R. J. \& Terlevich, E. 2003, ApJ, 598, 858

Matsuda, Y., Yamada, T., Hayashino, T., Yamauchi, R., \& Nakamura, Y. 2006, ApJ, 640, L123

Mo, H. J., \& White, S. D. M. 2002, MNRAS, 336, 112

Møller, P., Warren, S. J., Fall, S. M., Fynbo, J. U., \& Jakobsen, P. 2002, ApJ, 574,51

Murphy, M. T., \& Liske, J. 2004, MNRAS, 354, L31

Nagamine, K., Wolfe, A. M., Hernquist, L., \& Springel, V. 2007, ApJ, 660, 945

Neufeld, D. A. 1990, ApJ, 350, 216

Nilsson, K. K., Fynbo, J. P. U., Møller, P., Sommer-Larsen, J., \& Ledoux, C. 2006, A\&A, 452, L23

Nulsen, P. E. J., Barcons, X., \& Fabian, A. C. 1998, MNRAS, 301, 168

Ouchi, M., et al. 2008, ApJS, 176, 301

Peroux, C., Dessauges-Zavadsky, M., D’Odorico, S., Kim, T.-S., \& McMahon, R. G. 2005, MNRAS, 363, 479

Peroux, C., McMahon, R. G., Storrie-Lombardi, L. J., \& Irwin, M. J. 2003, MNRAS, 346, 1103

Prochaska, J. X., \& Wolfe, A. M. 1997, ApJ, 487, 73

Rao, S. M., Turnshek, D. A., \& Nestor, D. B. 2006, ApJ, 636, 610

Rauch, M., Haehnelt, M. G., \& Steinmetz, M. 1997a, ApJ, 481, 601

Rauch, M., et al. 1997b, ApJ, 489, 7

Schaye, J. 2001, ApJ, 559, L1

Scott, J. E., Kriss, G. A., Brotherton, M., Green, R. F., Hutchings, J., Shull, J. M., \& Zheng, W. 2004, ApJ, 615, 135

Shapley, A. E., Steidel, C. C., Pettini, M., \& Adelberger, K. L. 2003, ApJ, 588, 65

Simcoe, R. A., Sargent, W. L. W., \& Rauch, M. 2004, ApJ, 606, 92

Smith, D. J. B., \& Jarvis, M. J. 2007, MNRAS, 378, L49

Stark, D. P., Ellis, R. S., Richard, J., Kneib, J.-P., Smith, G. P., \& Santos, M. R. 2007, ApJ, 663, 10

Steidel, C. C. 1990, ApJS, 74, 37

Steidel, C. C., Adelberger, K. L., Giavalisco, M., Dickinson, M., \& Pettini, M. 1999, ApJ, 519, 1

Steidel, C. C., Adelberger, K. L., Shapley, A. E., Pettini, M., Dickinson, M., \& Giavalisco, M. 2000, ApJ, 532, 170

Steidel, C. C., \& Hamilton, D. 1993, AJ, 105, 2017

Stiavelli, M., Scarlata, C., Panagia, N., Treu, T., Bertin, G., \& Bertola, F. 2001, ApJ, 561, L37

Storrie-Lombardi, L. J., \& Wolfe, A. M. 2000, ApJ, 543, 552

Tapken, C., Appenzeller, I., Noll, S., Richling, S., Heidt, J., Meinköhn, E., \& Mehlert, D. 2007, A\&A, 467, 63

Tasitsiomi, A. 2006, ApJ, 645, 792

Telfer, R. C., Zheng, W., Kriss, G. A., \& Davidsen, A. F. 2002, ApJ, 565, 773

Tenorio-Tagle, G., Silich, S. A., Kunth, D., Terlevich, E., \& Terlevich, R. 1999, MNRAS, 309, 332

Trentham, N., Sampson, L., \& Banerji, M. 2005, MNRAS, 357, 783

van Breukelen, C., Jarvis, M. J., \& Venemans, B. P. 2005, MNRAS, 359, 895

Wang, L., Li, C., Kauffmann, G., \& De Lucia, G. 2007, MNRAS, 377, 1419

Warren, S. J., Møller, P., Fall, S. M., \& Jakobsen, P. 2001, MNRAS, 326, 759

Weatherley, S. J., Warren, S. J., Møller, P., Fall, S. M., Fynbo, J. U., \& Croom, S. M. 2005, MNRAS, 358, 985 
Weidinger, M. Møller, P., \& Fynbo, J. P. U. 2004, Nature, 430, 999

Weidinger, M., Møller, P., Fynbo, J. P. U., \& Thomsen, B. 2005, A\&A, 436, 825

Wild, V., Hewett, P. C., \& Pettini, M. 2007, MNRAS, 374, 292

Wolfe, A. M., \& Chen, H.-W. 2006, ApJ, 652, 981
Wolfe, A. M., Gawiser, E, \& Prochaska, J. X. 2003, ApJ, 593, 235

Wolfe, A. M., Lanzetta, K. M., Foltz, C. B., \& Chaffee, F. H. 1995, ApJ, 454, 698

Zheng, Z., \& Miralda-Escudé, J. 2002, ApJ, 578, 33 